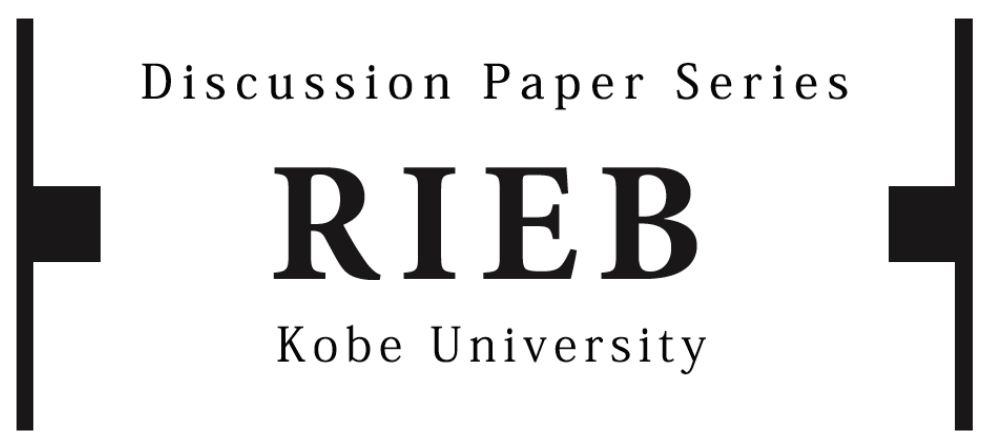

DP2010-04

\title{
Global Dynamics in Repeated Games with Additively Separable Payoffs
}

\author{
Takashi KAMIHIGASHI \\ Taiji FURUSAWA
}

Revised June 8, 2010

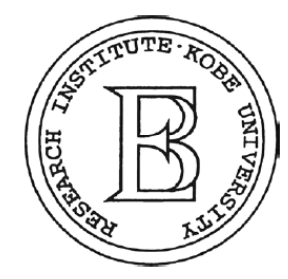

Research Institute for Economics and Business Administration Kobe University 


\title{
Global Dynamics in Repeated Games with Additively Separable Payoffs*
}

\author{
Takashi Kamihigashi ${ }^{\dagger}$ and Taiji Furusawa ${ }^{\ddagger}$
}

June 8, 2010

\begin{abstract}
This paper studies the global dynamics of a class of infinitely repeated two-player games in which the action space of each player is an interval, and the one-shot payoff of each player is additively separable in actions. We define an immediately reactive equilibrium (IRE) as a pure-strategy subgame perfect equilibrium such that each player's action is a stationary function of the opponent's last action. We completely characterize IREs and their dynamics in terms of certain indifference curves. Our results are used to show that in a prisoners' dilemma game with mixed strategies, gradual cooperation occurs when the players are sufficiently patient, and that in a certain duopoly game, kinked demand curves emerge naturally.
\end{abstract}

Keywords: Immediately reactive equilibria; additively separable payoffs; kinked demand; gradual cooperation; prisoners' dilemma

*(Forthcoming in the Review of Economic Dynamics) This paper has benefited considerably from the comments, suggestions, and criticisms made by an associate editor of the Review of Economic Dynamics and two anonymous referees. Earlier versions of this paper were presented in seminars at Kyoto University, University of Venice, University of Paris 1, and GREQAM. We would like to thank Atsushi Kajii, Tomoyuki Nakajima, Tadashi Sekiguchi, Olivier Tercieux, Julio Davila, Sergio Currarini, and Piero Gottardi for helpful comments and suggestions. We gratefully acknowledge financial support from the Japan Society for the Promotion of Science.

${ }^{\dagger}$ Corresponding author. RIEB, Kobe University, Rokkodai, Nada, Kobe 657-8501 JAPAN. Email: tkamihig@rieb.kobe-u.ac.jp. Tel/Fax: +81-78-803-7015.

${ }^{\ddagger}$ Graduate School of Economics, Hitotsubashi University, 2-1 Naka, Kunitachi, Tokyo 186-8601 JAPAN. Email: furusawa@econ.hit-u.ac.jp. Tel/Fax: +81-42-580-8866. 


\section{Introduction}

In infinitely repeated games with a prisoners' dilemma-like stage game, Nash reversion trigger strategies (Friedman, 1971) are often used to show that cooperation (or collusion) can be sustained by the threat to revert to a noncooperative Nash equilibrium. In such equilibria, each player continues to cooperate as long as all the other players do so, but will choose to behave selfishly once anyone defects. While Nash reversion equilibria are simple and intuitive, they seem to have two disadvantages. First, small deviations are punished as harshly as large deviations. Second, there are no nontrivial transition dynamics between cooperative and noncooperative states.

This paper studies the global dynamics of a class of infinitely repeated two-player games in which the action space of each player is an interval. We follow Friedman (1968) in focusing on strategies such that each player's action is a stationary function of the opponent's last action. We call such strategies immediately reactive, and say that a subgame perfect equilibrium is an immediately reactive equilibrium (IRE) if each player chooses an immediately reactive strategy. ${ }^{1}$ Unlike Nash reversion equilibria, IREs can be continuous; ${ }^{2}$ their global dynamics are typically nontrivial and can be characterized graphically.

In our framework, interesting dynamics arise naturally. For example, cooperation is achieved gradually in a repeated prisoners' dilemma with mixed strategies if the players are sufficiently patient. ${ }^{3}$ In a duopoly game, kinked demand curves emerge naturally. ${ }^{4}$ In a collusive steady state, each firm cuts its price if the other firm does so, but neither firm responds if the other firm

\footnotetext{
${ }^{1}$ Friedman (1968) called IREs "reaction function equilibria." We avoid his terminology since it has been used in a broader sense in the subsequent literature. The concept of IRE is related to a few other ones (Friedman and Samuelson, 1994a; Kalai, Samet, and Stanford, 1988; Maskin and Tirole, 1988a, 1988b); see Sections 2.3 and 2.4.

${ }^{2}$ Existence of nontrivial continuous equilibria has been studied by Samuelson (1987), Friedman and Samuelson (1990, 1994a, 1994b), and Langlois and Sachs (1993).

${ }^{3}$ Gradual cooperation is known to arise in certain partnership games (e.g., Kranton, 1996; Watson, 1999, 2002; Furusawa and Kawakami, 2006). Our example shows a simplest mechanism of gradual cooperation.

${ }^{4}$ Although there are game-theoretic models of kinked demand in the literature (e.g., Maskin and Tiroel, 1988b; Bhaskar, Machin, and Reid, 1991; Radner, 2003; Sen, 2004), they typically require rather specific assumptions. Though our example also requires specific assumptions, it allows one to derive and visualize kinked demand curves in an extremely simple manner.
} 
raises its price. In "inefficient" IREs, the collusive steady state is unstable: a small price cut by either firm triggers price war, eventually leading to a noncollusive steady state. In "efficient" IREs, however, the collusive steady state is stable: a price cut by either firm is matched by a smaller price cut, and the steady state is restored in the long run. All of these dynamic phenomena are properties of IREs in regular form, which we define as IREs that are continuous and punish deviations in a minimal way.

To obtain such sharp results, we assume that the one-shot payoff of each player is additively separable in actions. ${ }^{5}$ In addition we assume that each player's one-shot payoff is continuous, monotone in the opponent's action, and monotone or unimodal in his own action. These assumptions are satisfied in various games, including the models mentioned above.

As one can easily see, additive separability trivializes the analysis of oneshot games by ruling out strategic interactions. However, it does not trivialize the analysis of repeated games, where various interesting strategic interactions arise, as mentioned above. Those interactions are purely dynamic in nature since they are totally absent in one-shot games. In other words, our framework enables one to concentrate on purely dynamic phenomena.

Given a stage game satisfying the assumptions mentioned above, we show that the set of IREs in the simultaneous move game is identical to that in the alternating move game. ${ }^{6}$ In both games, we completely characterize IREs in terms of indifference curves associated with what we call effective payoffs. The effective payoff of a player is the part of his discounted sum of payoffs that is directly affected by his current action. ${ }^{7}$ We show that in any IRE, any equilibrium path stays on the associated indifference curves except for the initial period. By this result, equilibrium dynamics are always characterized by two indifference curves.

Our main results are as follows: First, a pair of indifference curves can be supported by an IRE if and only if it satisfies two certain graphical conditions.

\footnotetext{
${ }^{5}$ This assumption is also necessary since, as shown by Stanford (1986) and Robson (1986), the only possible IRE is a trivial one in certain duopoly games with additively non-separable payoffs.

${ }^{6}$ Section 2.6 discusses the relationship of this result to Lagunoff and Matsui's (1997) anti-folk theorem for alternating move games of pure coordination. See Haller and Lagunoff (2000) and Yoon (2001) for further results on alternating move games.

${ }^{7}$ Effective payoffs are similar to what Kamihigashi and Roy (2006) call partial gains in an optimal growth model with linear utility. Equations (2.13)-(2.15) in this paper are similar to (3.7)-(3.9) in Kamihigashi and Roy (2006), but essentially this is the only similarity in analysis between the two papers.
} 
Second, under these conditions, there is an IRE in regular form (which is continuous) supporting the given pair of indifference curves.

All our results depend critically on the simple observation that under additive separability, each player's dynamic maximization problem given the opponent's strategy reduces to a static problem. Although we fully exploit this special feature, it is only our point of departure. Our main results, which characterize the entire set of IREs in terms of effective payoffs, still require rather complex reasoning, as is evident in the proofs.

The rest of the paper is organized as follows. Section 2 describes the oneshot game and our assumptions, presents some examples, and introduces the simultaneous and the alternating move games. Section 3 discusses some immediate implications of the simple observation mentioned above. Section 4 examines the dynamics induced by IREs. Section 5 characterizes the entire set of IREs in terms of effective payoffs. Section 6 applies our results to a prisoner's dilemma and a duopoly game. Section 7 concludes the paper.

\section{The Games}

\subsection{The One-Shot Game}

Before introducing repeated games, we describe the underlying one-shot game. There are two players indexed by $i=1,2$. Each player's action space is given by $[0,1]$. This is merely a normalization, and each player's action space may be different, and may be any interval. Throughout we follow the following conventions unless otherwise indicated: (a) $i$ and $j$ always belong to $\{1,2\}$; (b) whenever $i$ (or $j$ ) is given, $j$ (or $i$ ) denotes the other index; (c) " $\forall i$ " means " $\forall i \in\{1,2\}$ ", " $\forall s_{i}$ " means " $\forall s_{i} \in[0,1]$," and these conventions also apply to other similar expressions.

Let $\pi_{i}:[0,1] \times[0,1] \rightarrow[-\infty, \infty)$ denote player $i$ 's payoff. The following assumptions are maintained throughout.

Assumption 2.1. For $i=1,2$, there exist $u_{i}:[0,1] \rightarrow[-\infty, \infty)$ and $v_{i}$ : $[0,1] \rightarrow \mathbb{R}$ such that

$$
\forall s_{i}, \forall s_{j}, \quad \pi_{i}\left(s_{i}, s_{j}\right)=u_{i}\left(s_{i}\right)+v_{i}\left(s_{j}\right) .
$$

Assumption 2.2. $v_{1}, v_{2}:[0,1] \rightarrow \mathbb{R}$ are continuous and bounded. Either both are strictly increasing or both are strictly decreasing. Without loss of 
generality we assume that both are strictly increasing, and we extend each $v_{i}$ to a strictly increasing continuous function from $\mathbb{R}$ to $\mathbb{R}$, denoted $v_{i}$ again, such that $\lim _{s \downarrow-\infty} v_{i}(s)=-\infty$ and $\lim _{s \uparrow \infty} v_{i}(s)=\infty$.

Assumption 2.3. For $i=1,2, u_{i}$ is continuous and bounded above. ${ }^{8}$ There exists $\hat{s}_{i} \in[0,1]$ such that $u_{i}$ is strictly increasing on $\left[0, \hat{s}_{i}\right]$ provided $\hat{s}_{i}>0$, and strictly decreasing on $\left[\hat{s}_{i}, 1\right]$ provided $\hat{s}_{i}<1$.

Assumption 2.1 is crucial to our analysis. Assumption 2.3 implies that $\hat{s}_{i}$ is player $i$ 's strictly dominant strategy and that $\left(\hat{s}_{1}, \hat{s}_{2}\right)$ is the unique static Nash equilibrium.

\subsection{Examples}

Although the above assumptions may appear rather restrictive, they are satisfied in various games. We provide specific examples below.

\subsubsection{Tariff War}

Consider a game between two countries, 1 and 2, with two goods, 1 and 2. Country $i$ exports good $i$, imports good $j$, and imposes a tariff rate $s_{i}$ on imports of good $j$. Let $v_{i}\left(s_{j}\right)$ denote the sum of country $i$ 's producer surplus and consumer surplus for good $i$. Under standard assumptions, $v_{i}$ is a strictly decreasing function of $s_{j}$. Let $u_{i}\left(s_{i}\right)$ denote the sum of country $i$ 's tariff revenue, consumer surplus, and producer surplus for good $j$. Optimal tariff theory suggests that $u_{i}$ is increasing where $s_{i}$ is small, and decreasing where $s_{i}$ is large. For simplicity we assume that $u_{i}$ has a single peak at $\hat{s}_{i}$, and that $u_{i}$ is strictly increasing for $s_{i} \leq \hat{s}_{i}$ and strictly decreasing for $s_{i} \geq \hat{s}_{i}$. Country $i$ seeks to maximize its welfare $u_{i}\left(s_{i}\right)+v_{i}\left(s_{j}\right)$. This game satisfies our assumptions, and is analyzed in detail in Furusawa and Kamihigashi (2006). ${ }^{9}$

\subsubsection{Duopoly}

Consider a game played by two firms. Each firm $i$ produces a differentiated product with a constant marginal $\operatorname{cost} c_{i}$ and with no fixed cost. Firm $i$

\footnotetext{
${ }^{8}$ We follow the convention that if $u_{i}(r)=-\infty$ for $r=0$ or 1 , then continuity of $u_{i}$ at $r$ means $\lim _{s \rightarrow r} u_{i}(s)=-\infty$.

${ }^{9} \mathrm{~A}$ preliminary version of Furusawa and Kamihigashi (2006) contained some of the arguments in this paper, which now appear exclusively in this paper.
} 
faces a demand function $D_{i}\left(p_{i}, p_{j}\right)$ that depends on the prices $p_{i}$ and $p_{j}$ chosen by the two firms. Firm $i$ 's profit is $D_{i}\left(p_{i}, p_{j}\right)\left(p_{i}-c_{i}\right)$. Suppose $D_{i}$ is multiplicatively separable: $D_{i}\left(p_{i}, p_{j}\right)=d_{i}^{i}\left(p_{i}\right) d_{i}^{j}\left(p_{j}\right)$ for some functions $d_{i}^{i}$ and $d_{i}^{j}$. Then the profit maximization problem of firm $i$ is equivalent to maximizing $u_{i}\left(p_{i}\right)+v_{i}\left(p_{j}\right)$, where

$$
u_{i}\left(p_{i}\right)=\ln d_{i}^{i}\left(p_{i}\right)+\ln \left(p_{i}-c_{i}\right), \quad v_{i}\left(p_{j}\right)=\ln d_{i}^{j}\left(p_{j}\right) .
$$

This transformation is innocuous in the one-shot game, and our assumptions are satisfied under reasonable assumptions on $d_{i}^{i}$ and $d_{i}^{j}$. In repeated games the above transformation may be justified by assuming that the owners of the firms are "risk averse," or prefer stable to unstable profit streams.

\subsubsection{Prisoner's Dilemma}

Though the action spaces are assumed to be intervals in this paper, our framework applies to $2 \times 2$ games with mixed strategies as well. A case in point is the prisoner's dilemma game in Figure 1 (with $a, c>0$ ), which is a parametrized version of the game discussed by Fudenberg and Tirole (1991, p. 10, p. 111). For $i=1,2$, let $s_{i}$ be player $i$ 's probability of choosing action C. Let $\pi_{i}\left(s_{i}, s_{j}\right)$ be player $i$ 's expected payoff:

$$
\begin{aligned}
\pi_{i}\left(s_{i}, s_{j}\right) & =s_{i} s_{j} c+s_{i}\left(1-s_{j}\right)(-a)+\left(1-s_{i}\right) s_{j}(c+a) \\
& =-a s_{i}+(c+a) s_{j} .
\end{aligned}
$$

Our assumptions are clearly satisfied with $\hat{s}_{1}=\hat{s}_{2}=0 .{ }^{10}$ In fact our assumptions are satisfied in more general $2 \times 2$ games as long as the coefficient of $s_{i} s_{j}$ is zero in $\pi_{i}\left(s_{i}, s_{j}\right)$.

\subsection{The Repeated Game with Simultaneous Moves}

Consider the infinitely repeated game in which the stage game is given by the one-shot game described in Section 2.1. For $i=1,2$, let $\delta_{i} \in(0,1)$ be player $i$ 's discount factor. We restrict ourselves to pure-strategy subgame perfect equilibria in which player $i$ 's action in period $t, s_{i, t}$, is a stationary function $f_{i}$ of player $j$ 's action in period $t-1, s_{j, t-1}$; i.e., $s_{i, t}=f_{i}\left(s_{j, t-1}\right)$. We call such strategies immediately reactive.

\footnotetext{
${ }^{10}$ Furusawa and Kawakami (2008) use a payoff function similar to (2.3) to analyze perfect Bayesian equilibria in a model with stochastic outside options.
} 
Player 2

\begin{tabular}{cc|c|c|}
\multicolumn{1}{c}{} & \multicolumn{1}{c}{$\mathrm{C}$} & \multicolumn{1}{c}{$\mathrm{D}$} \\
\cline { 3 - 4 } Player 1 & $\mathrm{C}$ & $c, c$ & $-a, c+a$ \\
\cline { 3 - 4 } & $\mathrm{D}$ & $c+a,-a$ & 0,0 \\
\cline { 3 - 4 } & & &
\end{tabular}

Figure 1: Prisoner's dilemma

Friedman (1968) called such strategies "reaction functions." Immediately reactive strategies are a special case of "single-period-recall strategies" (Friedman and Samuelson, 1994a) and "reactive strategies" (Kalai et al., 1988). Single-period-recall strategies depend only on both players' last actions, and reactive strategies depend only on the opponent's past actions. We focus on stationary strategies that depend only on the opponent's last action.

Let $F$ be the set of all functions from $[0,1]$ to $[0,1]$. Taking player $j$ 's strategy $f_{j} \in F$ as given, player $i$ faces the following problem:

$$
\begin{aligned}
\max _{\left\{s_{i, t}\right\}_{t=1}^{\infty}} & \sum_{t=1}^{\infty} \delta_{i}^{t-1}\left[u_{i}\left(s_{i, t}\right)+v_{i}\left(s_{j, t}\right)\right] \\
\text { s.t. } & \forall t \in \mathbb{N}, \quad s_{j, t}=f_{j}\left(s_{i, t-1}\right), \\
& \forall t \in \mathbb{N}, \quad s_{i, t} \in[0,1] .
\end{aligned}
$$

We say that $f_{i} \in F$ is a best response to $f_{j}$ if for any $\left(s_{i, 0}, s_{j, 0}\right) \in[0,1]^{2}$, the above maximization problem has a solution $\left\{s_{i, t}\right\}_{t=1}^{\infty}$ such that $s_{i, t}=f_{i}\left(s_{j, t-1}\right)$ for all $t \in \mathbb{N}$. We call a strategy profile $\left(f_{1}, f_{2}\right) \in F^{2}$ an immediately reactive equilibrium (IRE) if $f_{1}$ is a best response to $f_{2}$, and vice versa. Note that $f_{1}$ and $f_{2}$ are not required to be continuous or even measurable, but the maximization problem $(2.5)-(2.7)$ is required to be well defined given $f_{j}$. ${ }^{11}$

\footnotetext{
${ }^{11}$ Our results are unaffected even if $f_{1}$ and $f_{2}$ are required to be continuous or upper semi-continuous. The same remark applies to the alternating move game.
} 


\subsection{The Repeated Game with Alternating Moves}

Now consider the case of alternating moves. Player 1 updates his action in odd periods, while player 2 updates his action in even periods. ${ }^{12}$ Define

$$
T_{1}=\{1,3,5, \cdots\}, \quad T_{2}=\{2,4,6, \cdots\} .
$$

As in the simultaneous move case, we restrict ourselves to subgame perfect equilibria in which each player chooses an immediately reactive strategy, i.e, in each period $t \in T_{i}$, player $i$ chooses an action $s_{i, t}$ according to a stationary function $f_{i}$ of player $j$ 's last (or equivalently current) action $s_{j, t-1}$.

Given player $j$ 's strategy $f_{j} \in F$, player $i$ faces the following problem:

$$
\begin{aligned}
\max _{\left\{s_{i, t}\right\}_{t=i}^{\infty}} & \sum_{t=i}^{\infty} \delta_{i}^{t-i}\left[u_{i}\left(s_{i, t}\right)+v_{i}\left(s_{j, t}\right)\right] \\
\text { s.t. } & \forall t \in T_{j}, \quad s_{j, t}=f_{j}\left(s_{i, t-1}\right), s_{i, t}=s_{i, t-1}, \\
& \forall t \in T_{i}, \quad s_{i, t} \in[0,1], s_{j, t}=s_{j, t-1} .
\end{aligned}
$$

We say that $f_{i} \in F$ is a best response to $f_{j}$ if for any $s_{j, i-1} \in[0,1],{ }^{13}$ the above maximization problem has a solution $\left\{s_{i, t}\right\}_{t=i}^{\infty}$ such that $s_{i, t}=f_{i}\left(s_{j, t-1}\right)$ for all $t \in T_{i}$. We call a strategy profile $\left(f_{1}, f_{2}\right) \in F^{2}$ an immediately reactive equilibrium (IRE) if $f_{1}$ is a best response to $f_{2}$, and vice versa. This equilibrium concept is consistent with one definition of "Markov perfect equilibrium" (Maskin and Tirole, 1988b, Section 2), but distinct from another (Maskin and Tirole, 2001) due to additive separability of payoffs.

\subsection{Effective Payoffs}

We now introduce a function that plays a central role in our analysis. For $i=1,2$, define $w_{i}:[0,1]^{2} \rightarrow[-\infty, \infty)$ by

$$
w_{i}\left(s_{i}, s_{j}\right)=u_{i}\left(s_{i}\right)+\delta_{i} v_{i}\left(s_{j}\right) .
$$

We call this function player $i$ 's effective payoff since in both repeated games, player $i$ in effect seeks to maximize the discounted sum of effective payoffs.

\footnotetext{
${ }^{12}$ In alternating move games, it is often assumed that the players play simultaneously in the initial period and take turns afterwards. Such an assumption does not affect our analysis, which is concerned only with stationary subgame perfect equilibria.

${ }^{13}$ Notice that for $i=1,2$, the first period in which player $i$ plays is period $i$.
} 
Indeed, in both games, player $i$ 's discounted sum of payoffs from period 1 onward is written as

$$
\begin{aligned}
\sum_{t=1}^{\infty} \delta_{i}^{t-1}\left[v_{i}\left(s_{j, t}\right)+u_{i}\left(s_{i, t}\right)\right] & \\
= & v_{i}\left(s_{j, 1}\right)+\sum_{t=1}^{\infty} \delta_{i}^{t-1}\left[u_{i}\left(s_{i, t}\right)+\delta_{i} v_{i}\left(s_{j, t+1}\right)\right] \\
& =v_{i}\left(s_{j, 1}\right)+\sum_{t=1}^{\infty} \delta_{i}^{t-1} w_{i}\left(s_{i, t}, s_{j, t+1}\right)
\end{aligned}
$$

In both games, player $i$ has no influence on $s_{j, 1}$, so player $i$ 's problem is equivalent to maximizing the discounted sum of effective payoffs. This implies that each player's best responses are characterized by a static maximization problem:

Lemma 2.1. In both the simultaneous and the alternating move games, for $i=1,2, f_{i} \in F$ is a best response to $f_{j} \in F$ iff

$$
\forall s_{j}, \quad f_{i}\left(s_{j}\right) \in \underset{s_{i} \in[0,1]}{\operatorname{argmax}} w_{i}\left(s_{i}, f_{j}\left(s_{i}\right)\right) .
$$

In other words, $\left(f_{1}, f_{2}\right) \in F^{2}$ is an IRE iff (2.16) holds for $i=1,2$.

Proof. See Appendix A.

Lemma 2.1 implies that the simultaneous and the alternating move games are equivalent as far as IREs are concerned. This allows us to discuss IREs without specifying which repeated game is considered. The differences in dynamics between the two games are discussed in Section 4.

Given an $\operatorname{IRE}\left(f_{1}, f_{2}\right)$, we say that $\left(s_{1}, s_{2}\right) \in[0,1]^{2}$ is a steady state if $s_{1}=f_{1}\left(s_{2}\right)$ and $s_{2}=f_{2}\left(s_{1}\right)$. Needless to say, if the game starts from a steady state $\left(s_{1}, s_{2}\right)$, each player $i$ keeps choosing $s_{i}$ forever according to $f_{i}$. Note that any intersection of the graphs of $f_{1}$ and $f_{2}$ (on the $\left(s_{1}, s_{2}\right)$ plane) is a steady state.

\subsection{Discussions}

The equivalence between simultaneous and alternating move games discussed above would appear in sharp contrast to the anti-folk theorem of Lagunoff 
and Matsui (1997) for alternating move games of pure coordination. They show that there is a considerable difference between the simultaneous and the alternating move games in the case of pure coordination. If $u_{i}\left(s_{i}\right)=v_{j}\left(s_{i}\right)$ and $v_{i}\left(s_{j}\right)=u_{j}\left(s_{j}\right)$ for all $s_{i}, s_{j}$ and $i$, then the one-shot game described in Section 2.1 becomes a pure coordination game. Lemma 2.1 of course applies to this case (which is consistent with our assumptions), but does not contradict Lagunoff and Matsui's result. This is because their result deals with all subgame perfect equilibria, while Lemma 2.1 deals only with IREs.

One may also notice that the property of IREs given by (2.16) is similar to the definition of "conjectural variations equilibrium" (e.g., Figuières et al., 2004 , p. 14). The main difference is that a conjectural variations equilibrium consists of an action profile supported by "variational conjectures" as to how each player reacts to an infinitesimal deviation from the action profile by the opponent, while an IRE consists only of two functions that represent how each player optimally reacts to any action by the opponent.

To be more specific, let $\left(f_{1}, f_{2}\right) \in F^{2}$ be an IRE, and suppose that it has a steady state $\left(s_{1}, s_{2}\right) \in[0,1]^{2}$. Consider the one-shot game in which player $i$ 's payoff is given by $w_{i}\left(s_{i}, s_{j}\right)$. If both $f_{1}$ and $f_{2}$ are differentiable and $\left(s_{1}, s_{2}\right) \in(0,1)^{2}$, then $(2.16)$ implies that $\left(s_{1}, s_{2}\right)$ is a conjectural variations equilibrium with variational conjectures $\left(f_{1}^{\prime}, f_{2}^{\prime}\right)$ for this one-shot game. As we show in Section 5, however, IREs are typically not everywhere differentiable even when $u_{i}$ and $v_{i}$ are many times differentiable. Thus not every "interior" steady state can be supported as a conjectural variations equilibrium with variational conjectures $\left(f_{1}^{\prime}, f_{2}^{\prime}\right) .{ }^{14}$

On the other hand, any steady state of an IRE can be supported as a more general "conjectural equilibrium" (Figuières et al., 2004, p. 30), which does not require differentiability. In fact (2.16) is very similar to the definition of "consistent conjectural equilibrium." The main difference is that a consistent conjectural equilibrium is an action profile supported by "consistent conjectures," while an IRE consists only of two optimal reaction functions. ${ }^{15}$

\footnotetext{
${ }^{14}$ This does not mean that the set of steady states of IREs is larger than that of conjectural variations equilibria. On the contrary, under mild regularity conditions, any $\left(s_{1}, s_{2}\right) \in(0,1)^{2}$ is a conjectural variations equilibrium for the one-shot game considered here (Figuières et al., 2004, p. 14). Since Theorem 5.1 indicates that not every $\left(s_{1}, s_{2}\right) \in(0,1)^{2}$ can be supported as a steady state of an IRE, the set of steady states of IREs in $(0,1)^{2}$ is typically smaller than that of conjectural variations equilibria.

${ }^{15}$ See Sabourian (1992) and Figuières et al. (2004) for detailed discussions on the relation between conjectural variations equilibria and repeated games.
} 


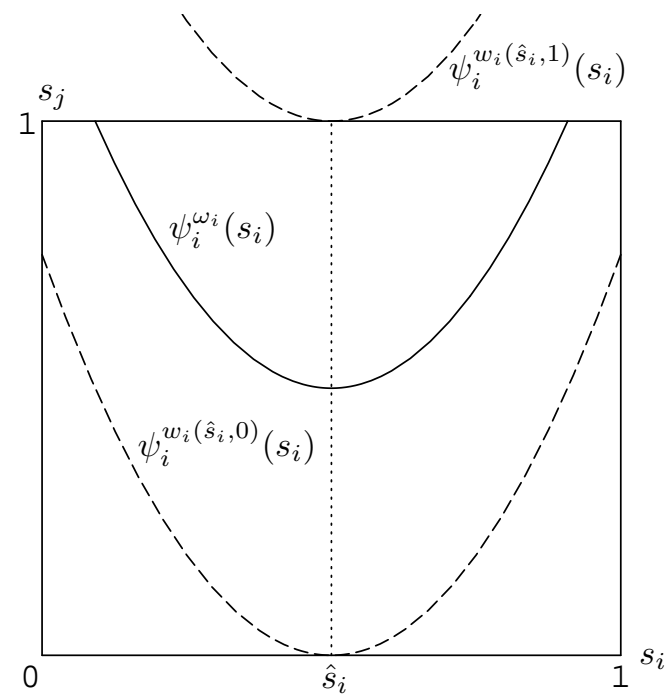

Figure 2: Indifference curve $\psi_{i}^{\omega_{i}}$ with $\omega_{i} \in\left[w_{i}\left(\hat{s}_{i}, 0\right), w_{i}\left(\hat{s}_{i}, 1\right)\right]$

\section{A Preliminary Characterization of IREs}

Lemma 2.1 suggests that the indifference curves associated with effective payoffs are closely related with best responses. Since each $v_{i}$ is strictly increasing and the inverse of $v_{i}$ is defined over $\mathbb{R}$ by Assumption 2.2, each indifference curve $w_{i}\left(s_{i}, s_{j}\right)=\omega_{i}$ can be expressed as the graph of a function from $s_{i}$ to $s_{j}$. We denote this function by $\psi_{i}^{\omega_{i}}$ :

$$
\forall s_{i}, \quad \omega_{i}=w_{i}\left(s_{i}, \psi_{i}^{\omega_{i}}\left(s_{i}\right)\right)=u_{i}\left(s_{i}\right)+\delta_{i} v_{i}\left(\psi_{i}^{\omega_{i}}\left(s_{i}\right)\right) .
$$

Equivalently,

$$
\forall s_{i}, \quad \psi_{i}^{\omega_{i}}\left(s_{i}\right)=v_{i}^{-1}\left(\frac{\omega_{i}-u_{i}\left(s_{i}\right)}{\delta_{i}}\right) .
$$

Since $v_{i}$ is strictly increasing, a higher indifference curve is associated with a higher effective payoff. See Figure 2.

Given player $j$ 's strategy $f_{j} \in F$, let $\left.\omega_{i}^{*}\right|_{f_{j}}$ denote player $i$ 's maximum effective payoff:

$$
\left.\omega_{i}^{*}\right|_{f_{j}}=\sup _{s_{i} \in[0,1]} w_{i}\left(s_{i}, f_{j}\left(s_{i}\right)\right) .
$$

Assuming that the supremum is attained at some $s_{i}$, the associated maxi- 
mization problem can be expressed as

$$
\max _{s_{i}, s_{j} \in[0,1]} w_{i}\left(s_{i}, s_{j}\right) \quad \text { s.t. } s_{j}=f_{j}\left(s_{i}\right) \text {. }
$$

Let $\left.\psi_{i}^{*}\right|_{f_{j}}$ be player $i$ 's optimal indifference curve given player $j$ 's strategy $f_{j}$ :

$$
\left.\psi_{i}^{*}\right|_{f_{j}}=\psi_{i}^{\left.\omega_{i}^{*}\right|_{f_{j}}}
$$

Note that in (3.4), player $i$ takes $s_{j}=f_{j}\left(s_{i}\right)$ as a constraint. Thus $\left.\psi_{i}^{*}\right|_{f_{j}}$ is the highest indifference curve tangent to the graph of $f_{j}$. See Figure 3.

The following result provides a necessary and sufficient condition for a given strategy profile to be an IRE:

Proposition 3.1. A strategy profile $\left(f_{1}, f_{2}\right) \in F^{2}$ is an IRE iff

$$
\forall i, \forall s_{j}, \quad f_{j}\left(f_{i}\left(s_{j}\right)\right)=\left.\psi_{i}^{*}\right|_{f_{j}}\left(f_{i}\left(s_{j}\right)\right)
$$

Proof. For sufficiency, assume (3.6). Fix $i$ and $s_{j}$. Let $s_{i}=f_{i}\left(s_{j}\right)$. Then $w_{i}\left(s_{i}, f_{j}\left(s_{i}\right)\right)=w_{i}\left(s_{i},\left.\psi_{i}^{*}\right|_{f_{j}}\left(s_{i}\right)\right)=\left.\omega_{i}^{*}\right|_{f_{j}}$. Thus by Lemma 2.1, $f_{i}$ is a best response to $f_{j}$. It follows that $\left(f_{1}, f_{2}\right)$ is an IRE. Reversing this argument yields the converse.

To illustrate Proposition 3.1, let $\left(f_{1}, f_{2}\right)$ be given by $f_{i}\left(s_{j}\right)=\hat{s}_{i}$ for all $s_{j}$ and $i$. In other words each player plays his static Nash strategy. See Figure 4. One can easily see that $\left(f_{1}, f_{2}\right)$ satisfies (3.6), so it is an IRE.

As another example, let $\left(f_{1}, f_{2}\right)$ be such that $f_{i}\left(s_{j}\right)=\bar{s}_{i}$ if $s_{j}=\bar{s}_{j}$, and $f_{i}\left(s_{j}\right)=\hat{s}_{i}$ otherwise, where $\bar{s}_{1}$ and $\bar{s}_{2}$ are as in Figure 5 . In this case each player "cooperates" as long as the opponent does so, but reverts to the static Nash strategy if the opponent deviates at all. Once again one can easily see that $\left(f_{1}, f_{2}\right)$ satisfies (3.6), so it is an IRE.

While Proposition 3.1 can be used to construct specific IREs as above, or to check whether a given strategy profile is an IRE, it does not provide the entire picture of how large the set of IREs may be. The main purpose of this paper is to characterize the entire set of IREs. To this end we define an IRE associated with $\omega=\left(\omega_{1}, \omega_{2}\right) \in \mathbb{R}^{2}$ as an $\operatorname{IRE}\left(f_{1}, f_{2}\right)$ such that

$$
\forall i, \quad \omega_{i}=\left.\omega_{i}^{*}\right|_{f_{j}}
$$

\footnotetext{
${ }^{16}$ From here on we omit the arguments of functions in figures.
} 


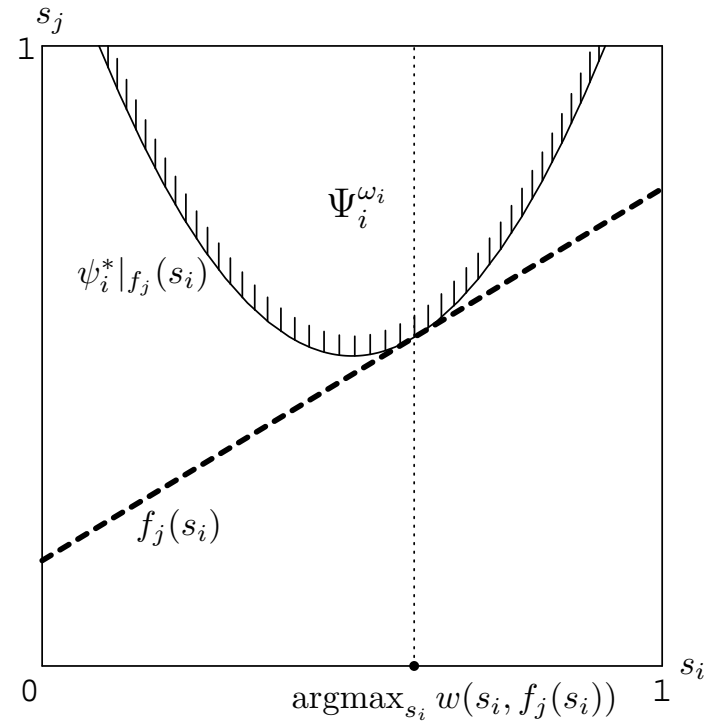

(a)

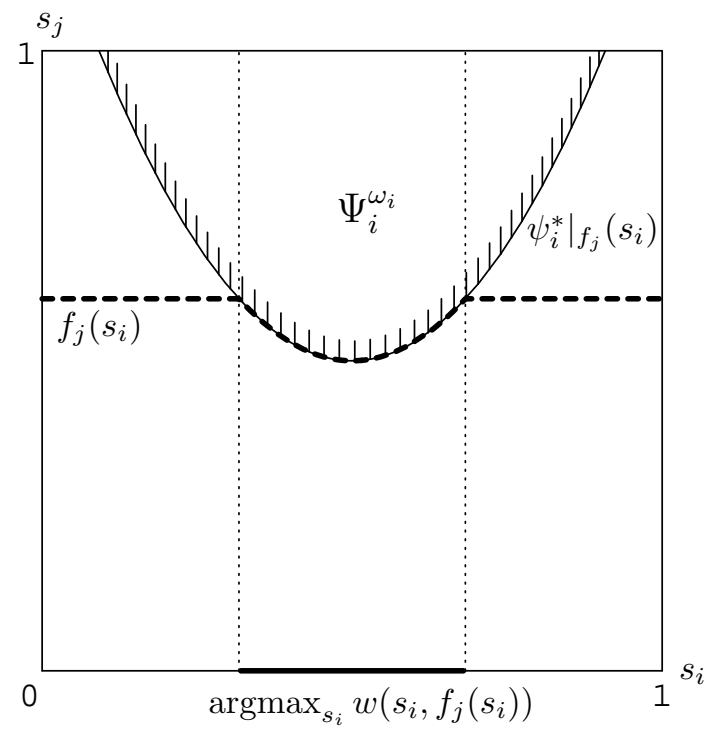

(b)

Figure 3: $\left.\psi_{i}^{*}\right|_{f_{j}}$ and $\Psi_{i}^{\omega_{i}}$ (defined in (5.1)) 


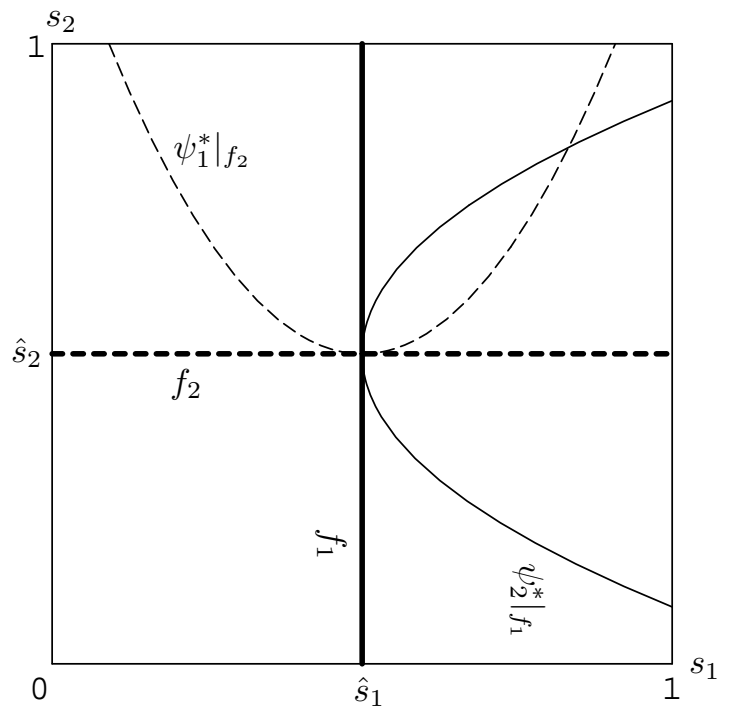

Figure 4: Static Nash equilibrium ${ }^{16}$

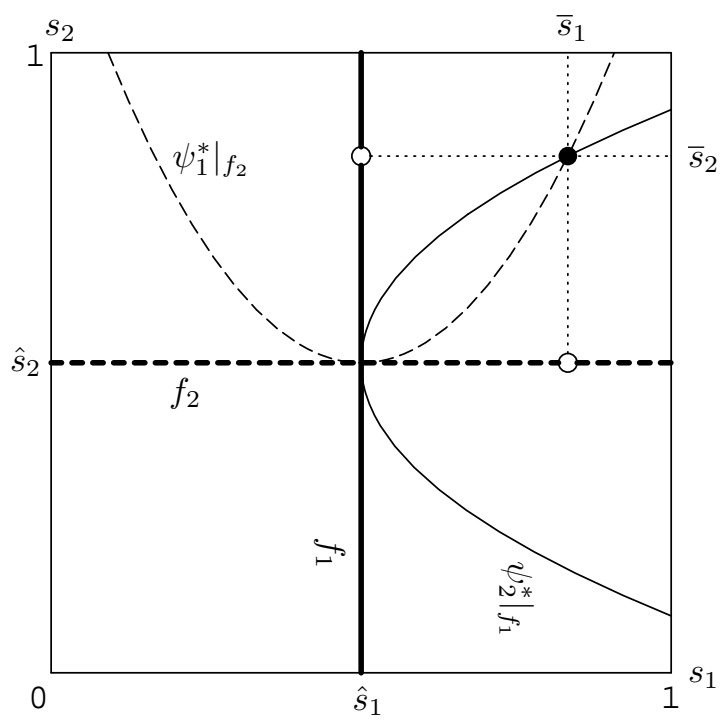

Figure 5: Nash reversion 
In other words, in an IRE associated with $\omega$, each player $i$ 's effective payoff is $\omega_{i}$. Notice that any $\operatorname{IRE}\left(f_{1}, f_{2}\right)$ is associated with $\left(\left.\omega_{1}^{*}\right|_{f_{2}},\left.\omega_{2}^{*}\right|_{f_{1}}\right)$ by definition. The following result shows one way to construct a nontrivial IRE associated with $\omega \in \mathbb{R}^{2}$.

Proposition 3.2. Let $\omega \in \mathbb{R}^{2}$. Suppose

$$
\forall i, \forall s_{i}, \quad \psi_{i}^{\omega_{i}}\left(s_{i}\right) \in[0,1]
$$

Then $\left(f_{1}, f_{2}\right)=\left(\psi_{2}^{\omega_{2}}, \psi_{1}^{\omega_{1}}\right)$ is an IRE associated with $\omega$.

Proof. Let $\left(f_{1}, f_{2}\right)=\left(\psi_{2}^{\omega_{2}}, \psi_{1}^{\omega_{1}}\right)$. Then for each $i$,

$$
\forall s_{i}, \quad w_{i}\left(s_{i}, f_{j}\left(s_{i}\right)\right)=w_{i}\left(s_{i}, \psi_{i}^{\omega_{i}}\left(s_{i}\right)\right)=\omega_{i},
$$

so (3.7) trivially holds. ${ }^{17}$ It follows that $\left.\psi_{i}^{*}\right|_{f_{j}}=\psi_{i}^{\omega_{i}}=f_{j}$ for both $i$. Thus (3.6) holds, and $\left(f_{1}, f_{2}\right)$ is an IRE by Proposition 3.1.

This result can be seen more directly as follows: Notice that if $\left(f_{1}, f_{2}\right)=$ $\left(\psi_{2}^{\omega_{2}}, \psi_{1}^{\omega_{1}}\right)$, each player is entirely indifferent among all possible actions, since whatever player $i$ does, his effective payoff is $\omega_{i}$ by (3.9). This implies that both players are entirely indifferent among all possible strategies; in particular, choosing $f_{i}=\psi_{j}^{\omega_{j}}$ is optimal for each player $i$, which makes $\left(f_{1}, f_{2}\right)$ an IRE. See Figure 6 for an example of an IRE satisfying (3.8) and (3.7).

\section{Dynamics}

Before we turn to our main results, it is useful to have a basic understanding of the dynamics induced by IREs. For this purpose we take an $\operatorname{IRE}\left(f_{1}, f_{2}\right) \in$ $F^{2}$ as given and study its dynamic properties in this section.

Consider first the alternating move game. Recall that in each period $t \in \mathbb{N}$, player $i$ with $t \in T_{i}$ updates his action as a function of player $j$ 's last (or current) action. So the "state variable" in each period $t \in T_{i}$ is player $j$ 's last action $s_{j, t-1}$. Given initial condition $s_{2,0}$, the entire path $\left\{s_{1, t}, s_{2, t}\right\}_{t=1}^{\infty}$ (with $s_{2,1}=s_{2,0}$ ) of the game is uniquely determined by

$$
\forall i, \forall t \in T_{i}, \quad s_{i, t+1}=s_{i, t}=f_{i}\left(s_{j, t-1}\right) .
$$

\footnotetext{
${ }^{17}$ Note that (3.9) implies $\sup _{s_{i}} w_{i}\left(s_{i}, f_{j}\left(s_{i}\right)\right)=\omega_{i}$.
} 


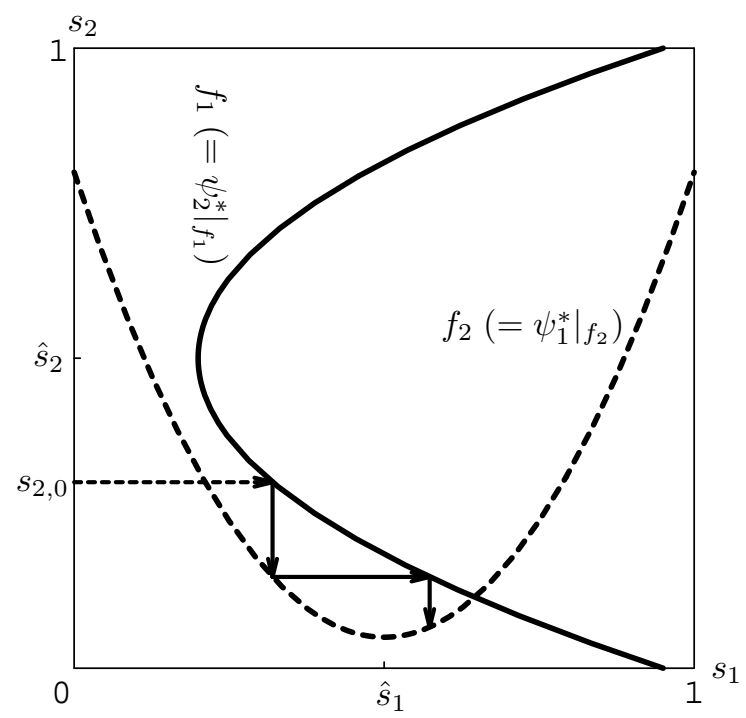

Figure 6: Example of IRE satisfying (3.8) and (3.7).

This means that

$$
s_{1,1}=f_{1}\left(s_{2,0}\right), s_{2,2}=f_{2}\left(s_{1,1}\right), s_{1,3}=f_{1}\left(s_{2,2}\right), \cdots .
$$

For the alternating move game, we define an IRE path associated with $\left(f_{1}, f_{2}\right)$ as a sequence $\left\{s_{1, t}, s_{2, t}\right\}_{t=0}^{\infty}$ satisfying (4.1) and $s_{2,1}=s_{2,0} \cdot{ }^{18}$ See Figure 6 for an example of an IRE path.

Now consider the simultaneous move game. The state variable in each period $t \in \mathbb{N}$ is the pair of both players' last actions $\left(s_{1, t-1}, s_{2, t-1}\right)$. Given initial condition $\left(s_{1,0}, s_{2,0}\right)$, the entire path $\left\{s_{1, t}, s_{2, t}\right\}_{t=1}^{\infty}$ of the game is uniquely determined by

$$
\forall i, \forall t \in \mathbb{N}, \quad s_{i, t}=f_{i}\left(s_{j, t-1}\right) .
$$

For the simultaneous move game, we define an IRE path associated with $\left(f_{1}, f_{2}\right)$ as a sequence $\left\{s_{1, t}, s_{2, t}\right\}_{t=0}^{\infty}$ satisfying (4.3). Any IRE path can be decoupled into two sequences, one originating from $s_{2,0}$, the other from $s_{1,0}$ :

$$
\begin{aligned}
& s_{1,1}=f_{1}\left(s_{2,0}\right), s_{2,2}=f_{2}\left(s_{1,1}\right), s_{1,3}=f_{1}\left(s_{2,2}\right), \cdots, \\
& s_{2,1}=f_{2}\left(s_{1,0}\right), s_{1,2}=f_{1}\left(s_{2,1}\right), s_{2,3}=f_{2}\left(s_{1,2}\right), \cdots .
\end{aligned}
$$

\footnotetext{
${ }^{18}$ Though $s_{1,0}$ is irrelevant for this game, it is included here for notational simplicity.
} 
Obviously, given $s_{2,0}$, the sequences given by (4.2) and (4.4) are identical. The sequence given by (4.5) can be viewed as an IRE path for the alternating move game in which player 2 moves first. Hence an IRE path for the simultaneous move game is equivalent to a pair of IRE paths for the two alternating move games in one of which player 1 moves first and in the other player 2 moves first.

The following result is a simple consequence of Proposition 3.1.

Theorem 4.1. Any IRE path $\left\{s_{1, t}, s_{2, t}\right\}_{t=0}^{\infty}$ associated with $\left(f_{1}, f_{2}\right) \in F^{2}$ for the simultaneous move game satisfies

$$
\forall t \geq 2, \forall i, \quad s_{i, t}=\left.\psi_{j}^{*}\right|_{f_{i}}\left(s_{j, t-1}\right) .
$$

Furthermore, any IRE path $\left\{s_{1, t}, s_{2, t}\right\}_{t=0}^{\infty}$ associated with $\left(f_{1}, f_{2}\right) \in F^{2}$ for the alternating move game satisfies

$$
\forall t \geq 2, \forall i, \quad t \in T_{i} \Rightarrow s_{i, t}=\left.\psi_{j}^{*}\right|_{f_{i}}\left(s_{j, t-1}\right) .
$$

Proof. Consider the simultaneous move game. Let $\left\{s_{1, t}, s_{2, t}\right\}_{t=0}^{\infty}$ be an IRE path associated with $\left(f_{1}, f_{2}\right)$. For each $i$ and $t \geq 2$, we have

$$
s_{i, t}=f_{i}\left(s_{j, t-1}\right)=f_{i}\left(f_{j}\left(s_{i, t-2}\right)\right)=\left.\psi_{j}^{*}\right|_{f_{i}}\left(f_{j}\left(s_{i, t-2}\right)\right)=\left.\psi_{j}^{*}\right|_{f_{i}}\left(s_{j, t-1}\right),
$$

where the third equality uses (3.6) (with $i$ and $j$ interchanged). Now (4.6) follows. The proof for the alternating move game is similar.

The above result shows that any IRE path is characterized by the corresponding pair of indifference curves $\left(\left.\psi_{2}^{*}\right|_{f_{1}},\left.\psi_{1}^{*}\right|_{f_{2}}\right)$ except for the initial period. To better understand this result, consider the alternating move game. The initial period must be excluded in (4.7) because $s_{2,0}$ is an arbitrary initial condition that need not be optimal for player 2 given $f_{1}$, i.e., it need not satisfy $s_{1,1}=\left.\psi_{2}^{*}\right|_{f_{1}}\left(s_{2,0}\right)$. Since all subsequent actions must be individually optimal, they must be on the optimal indifference curves. In Figure 6, any IRE path satisfies the equality in (4.7) for all $t \geq 1$. In Figure 4 , by contrast, an IRE path (not shown in the figure) violates the equality for $t=1$ unless $s_{2,0}=\hat{s}_{2}$, but trivially satisfies it for $t \geq 2$.

Theorem 4.1 also shows that in both games the dynamics of an IRE associated with $\omega \in \mathbb{R}^{2}$ are essentially characterized by the same dynamical system:

$$
\forall t \in T_{1}, \quad s_{1, t+2}=\psi_{2}^{\omega_{2}}\left(\psi_{1}^{\omega_{1}}\left(s_{1, t}\right)\right) .
$$


To be precise, the simultaneous move game has another equation, $s_{2, t+2}=$ $\psi_{1}^{\omega_{1}}\left(\psi_{2}^{\omega_{2}}\left(s_{2, t}\right)\right)$ for $t \in T_{1}$, but this system is equivalent to (4.9) in terms of dynamics. Hence one can obtain conditions for dynamic properties such as monotonicity and chaos by applying numerous results available on onedimensional dynamical systems (e.g., Devaney, 1989). ${ }^{19}$

\section{Main Results}

Theorem 4.1 shows that the dynamics of an IRE are characterized by the associated pair of indifference curves. The remaining question then is what pairs of indifference curves are supported by IREs. This section answers this question.

For $i=1,2$ and $\omega \in \mathbb{R}^{2}$, define

$$
\begin{aligned}
\Psi_{i}^{\omega_{i}} & =\left\{\left(s_{i}, s_{j}\right) \in[0,1]^{2} \mid w_{i}\left(s_{i}, s_{j}\right) \geq \omega_{i}\right\} \\
& =\left\{\left(s_{i}, s_{j}\right) \in[0,1]^{2} \mid \psi_{i}^{\omega_{i}}\left(s_{i}\right) \leq s_{j}\right\} .
\end{aligned}
$$

The set $\Psi_{i}^{\omega_{i}}$ is the collection of all pairs $\left(s_{i}, s_{j}\right)$ with player $i$ 's effective payoff at least as large as $\omega_{i}$. On the $\left(s_{i}, s_{j}\right)$ plane, it is the area on or above the graph $s_{j}=\psi_{i}^{\omega_{i}}\left(s_{i}\right)$; see Figure 3 . Provided $\Psi_{i}^{\omega_{i}} \cap \Psi_{j}^{\omega_{j}} \neq \emptyset,{ }^{20}$ we define

$$
\begin{aligned}
\bar{s}_{i}^{\omega} & =\max \left\{s_{i} \in[0,1] \mid \exists s_{j} \in[0,1],\left(s_{i}, s_{j}\right) \in \Psi_{i}^{\omega_{i}} \cap \Psi_{j}^{\omega_{j}}\right\}, \\
\underline{s}_{i}^{\omega} & =\min \left\{s_{i} \in[0,1] \mid \psi_{i}^{\omega_{i}}\left(s_{i}\right) \leq \bar{s}_{j}^{\omega}\right\} .
\end{aligned}
$$

See Figure 7. Note from (5.3) and (5.2) that there is $s_{i}$ such that $\psi_{i}^{\omega_{i}}\left(s_{i}\right) \leq \bar{s}_{j}^{\omega}$ and $\psi_{j}^{\omega_{j}}\left(\bar{s}_{j}^{\omega}\right) \leq s_{i}$; the former inequality assures the existence of $\underline{s}_{i}^{\omega}$. In Figure $6, \bar{s}_{1}^{\omega}=\bar{s}_{2}^{\omega}=1$ and $\underline{s}_{1}^{\omega}=\underline{s}_{2}^{\omega}=0$.

We are now ready to state our main results.

Theorem 5.1. Let $\omega \in \mathbb{R}^{2}$. There exists an IRE associated with $\omega$ iff

$$
\begin{gathered}
\Psi_{1}^{\omega_{1}} \cap \Psi_{2}^{\omega_{2}} \neq \emptyset \\
\forall i, \quad \underline{s}_{i}^{\omega} \leq \psi_{j}^{\omega_{j}}\left(\hat{s}_{j}\right) .
\end{gathered}
$$

\footnotetext{
${ }^{19}$ See Rand (1978) for an early example of complex dynamics in an "adaptive" dynamic model that has a structure similar to Figure 6. See Rosser (2002) for a recent survey of adaptive duopoly/oligopoly models that generate complex dynamics.

${ }^{20} \mathrm{It}$ is understood that the coordinates of $\Psi_{j}^{\omega_{j}}$ (or $\Psi_{i}^{\omega_{i}}$ ) are interchanged so that $\Psi_{i}^{\omega_{i}}$ and $\Psi_{j}^{\omega_{j}}$ have the same order of coordinates. Similar comments apply to similar expressions below.
} 


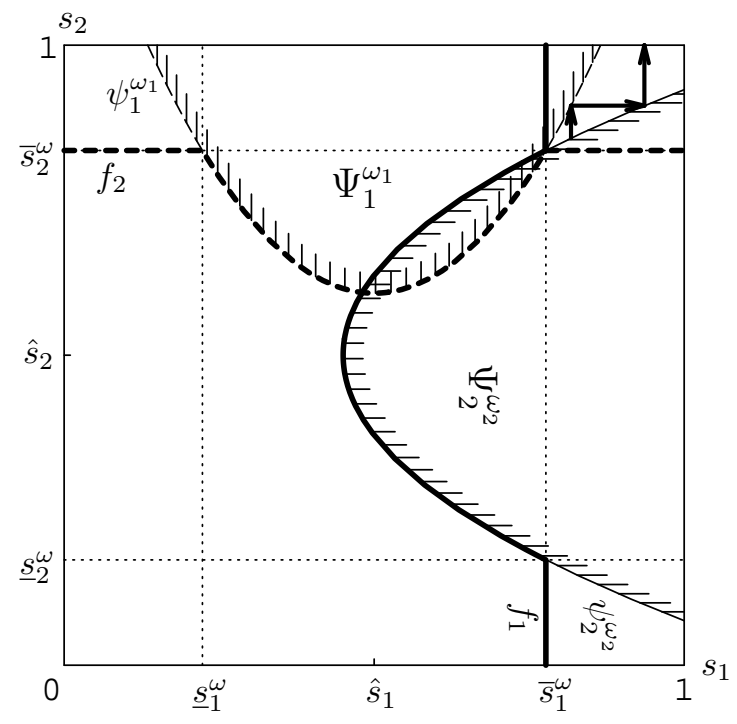

Figure $7: \underline{s}_{i}, \bar{s}_{i}$, and IRE in regular form

Proof. See Appendix B.

The sufficiency part of the above result is a consequence of the following:

Theorem 5.2. ${ }^{21}$ Let $\omega \in \mathbb{R}^{2}$ satisfy (5.5) and (5.6). Define $\left(f_{1}, f_{2}\right) \in F^{2}$ by

$$
\forall s_{j}, \quad f_{i}\left(s_{j}\right)=\min \left\{\psi_{j}^{\omega_{j}}\left(s_{j}\right), \bar{s}_{i}^{\omega}\right\} .
$$

Then $\left(f_{1}, f_{2}\right)$ is an IRE associated with $\omega .^{22}$ Furthermore, there exists $\left(s_{1}, s_{2}\right) \in$ $[0,1]^{2}$ such that

$$
\forall i, \quad\left(\text { a) } \omega_{i}=w_{i}\left(s_{i}, s_{j}\right), \quad \text { (b) } s_{i}=f_{i}\left(s_{j}\right)=\psi_{j}^{\omega_{j}}\left(s_{j}\right) .\right.
$$

Proof. See Appendix B.1.

We say that an IRE satisfying (5.7) is in regular form. Note that any IRE in regular form is continuous (i.e, each $f_{i}$ is continuous). See Figure 7 for an example of an IRE in regular form. By (5.8), any IRE in regular form has a steady state $\left(s_{i}, s_{j}\right)$, where the two indifference curves cross each other.

\footnotetext{
${ }^{21}$ We would like to thank an anonymous referee for his or her suggestion to emphasize this result.

${ }^{22}$ By Lemma B.2, $\psi_{j}^{\omega_{j}}\left(\hat{s}_{j}\right) \leq \bar{s}_{i}^{\omega}$, so $f_{i}\left(\hat{s}_{j}\right)=\psi_{j}^{\omega_{j}}\left(\hat{s}_{j}\right)$.
} 
We call (5.5) the nonemptiness condition, and (5.6) the no-sticking-out condition. Theorem 5.1 shows that these conditions are necessary and sufficient for an IRE associated with $\omega \in \mathbb{R}^{2}$ to exist. The nonemptiness condition says that the intersection of the two sets $\Psi_{1}^{\omega_{1}}$ and $\Psi_{2}^{\omega_{2}}$ must be nonempty. The no-sticking-out condition says that the graph of $\psi_{j}^{\omega_{j}}$ must not "stick out" of the straight line $s_{i}=\underline{s}_{i}$.

These conditions can be better understood by considering examples where they are violated. In Figure 8(a), the nonemptiness condition (5.5) is violated. In this case, any IRE path for the alternating move game must behave like the path depicted in the figure (except for the initial period) by Theorem 4.1. But since such a path cannot stay on the indifference curves forever, it cannot be an IRE path. In Figure 8(b), the no-sticking-out condition (5.6) is violated for $i=1$. In this case, if an IRE associated with $\omega \in \mathbb{R}^{2}$ exists, there is $s_{2,0}$ such that $f_{1}\left(s_{2,0}\right) \leq \psi_{2}^{\omega_{2}}\left(s_{2,0}\right)<\underline{s}_{1}^{\omega} \cdot{ }^{23}$ As shown in the figure, the IRE path from such $s_{2,0}$ cannot stay on the indifference curves forever.

We should mention that the IRE in regular form associated with $\omega \in$ $\mathbb{R}^{2}$ is not the only IRE associated with $\omega$. However, any IRE satisfies one restriction:

Proposition 5.1. Let $\left(f_{1}, f_{2}\right)$ be an IRE associated with $\omega \in \mathbb{R}^{2}$. Then

$$
\forall i, \forall s_{j}, \quad f_{i}\left(s_{j}\right) \leq \bar{s}_{i}^{\omega} .
$$

Proof. Immediate from (B.14) and (B.26).

To see the idea of this result, suppose the inequality in (5.9) is violated for $i=1$. Consider the alternating move game. Then for some $s_{2,0}$, we have $s_{1,1}=f_{1}\left(s_{2,0}\right)>\bar{s}_{1}^{\omega}$. If this path is continued, it behaves like the one depicted in Figure 7 by Theorem 4.1. But such a path cannot be an IRE path since it cannot stay on the indifference curves forever.

Proposition 5.1 along with (5.7) implies that if $\left(f_{1}, f_{2}\right)$ is an IRE associated with $\omega \in \mathbb{R}^{2}$, and if $\left(\bar{f}_{1}, \bar{f}_{2}\right)$ is the IRE in regular form associated with $\omega$, then $f_{i} \leq \bar{f}_{i}$ for both $i$. In other words, in the IRE $\left(\bar{f}_{1}, \bar{f}_{2}\right)$, each player gives the opponent the highest possible effective payoff among all IREs associated with $\omega$ in response to any action by the opponent.

In what follows, we say that an IRE $\left(f_{1}, f_{2}\right)$ is effectively efficient if there is no $\operatorname{IRE}\left(\tilde{f}_{1}, \tilde{f}_{2}\right)$ such that $\left.\omega_{1}^{*}\right|_{f_{2}} \leq\left.\omega_{1}^{*}\right|_{\tilde{f}_{2}}$ and $\left.\omega_{2}^{*}\right|_{f_{1}} \leq\left.\omega_{2}^{*}\right|_{\tilde{f}_{1}}$ with at least

\footnotetext{
${ }^{23}$ The first inequality holds since $\psi_{2}^{\omega_{2}}=\left.\psi_{2}^{*}\right|_{f_{1}}$; recall Figure 3. Figure 8(b) implicitly assumes $f_{1}\left(s_{2,0}\right)=\psi_{2}^{\omega_{2}}\left(s_{2,0}\right)$.
} 


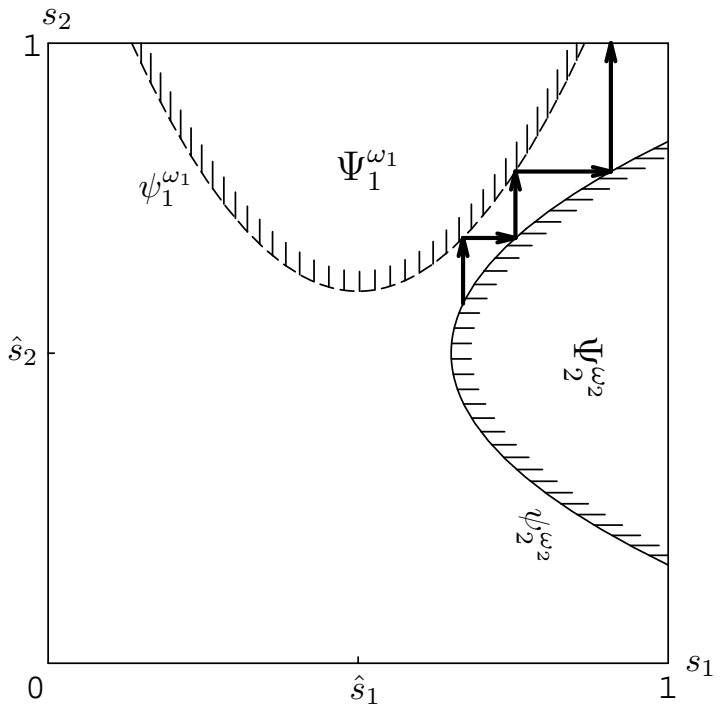

(a)

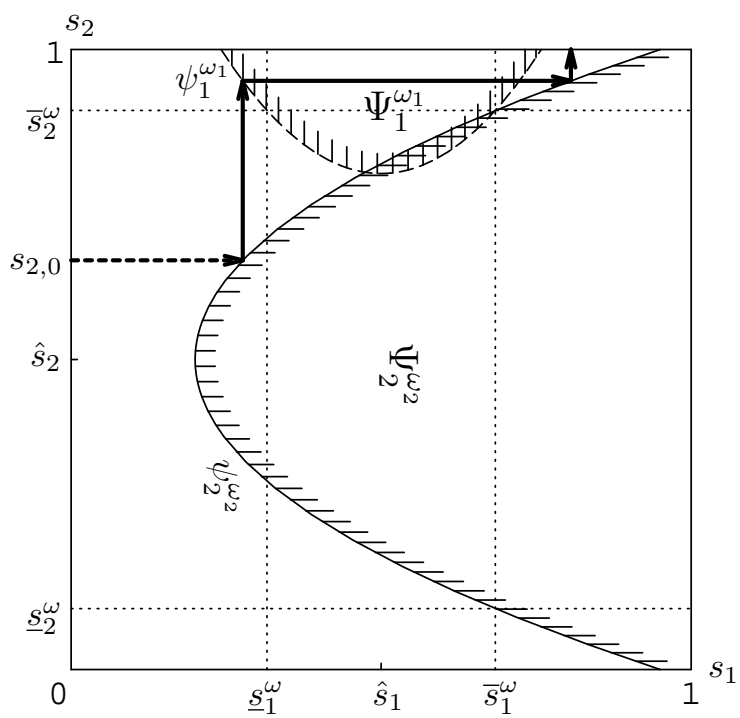

(b)

Figure 8: Examples with no IRE 


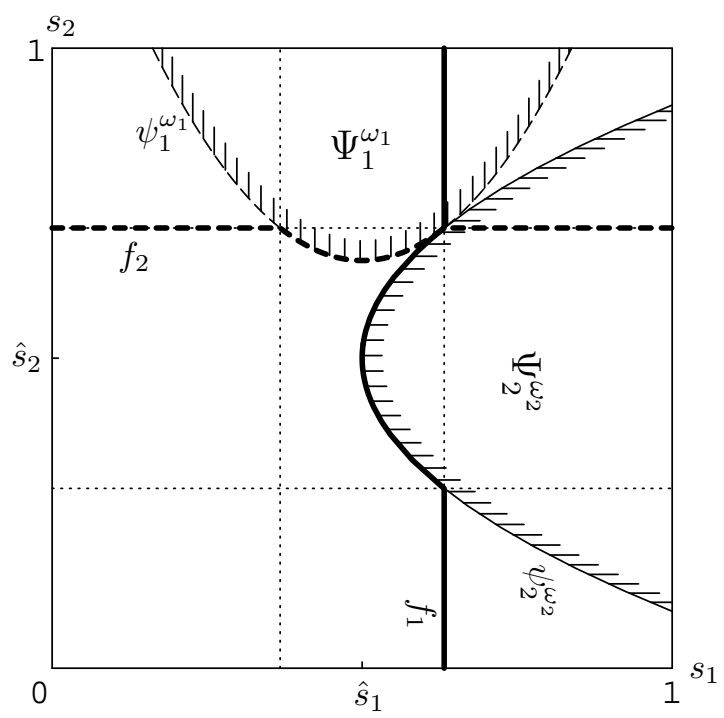

Figure 9: Effectively efficient IRE

one of the inequalities holding strictly. That is, $\left(f_{1}, f_{2}\right)$ is effectively efficient if it is not Pareto dominated by any other IRE in terms of effective payoffs. As illustrated in Section 6, effective efficiency has important dynamic implications.

For $i=1,2$ and $\omega \in \mathbb{R}^{2}$, define

$$
\tilde{\Psi}_{i}^{\omega_{i}}=\left\{\left(s_{i}, s_{j}\right) \in[0,1]^{2} \mid w_{i}\left(s_{i}, s_{j}\right)>\omega_{i}\right\} .
$$

Since $\psi_{i}^{\omega_{i}}$ is strictly increasing in $\omega_{i}$, it is clear from Theorem 5.1 that an IRE associated with $\omega$ is effectively efficient if

$$
\tilde{\Psi}_{1}^{\omega_{1}} \cap \tilde{\Psi}_{2}^{\omega_{2}}=\emptyset .
$$

See Figures 9 and 8(a).

One might conjecture that (5.11) is also necessary for effective efficiency. Unfortunately it is not the case. This is because the no-sticking-out condition (5.6), a necessary condition for an IRE, is not stable under small perturbations to $\omega$. In other words, even when (5.11) does not hold, (5.6) can be violated if $\omega$ is slightly changed. For example, when (5.6) holds with equality for $i=1$, it can be violated after $\omega_{2}$ is slightly increased, depending on how fast the two sides of the inequality in (5.6) vary with $\omega_{2}$. 
Even if (5.6) holds with strict inequality, it can be violated after small perturbations to $\omega$, since $\underline{s}_{i}^{\omega}$ need not be continuous in $\omega$. Figure 10 illustrates this point. There is an IRE in Figure 10(a), but there is no IRE in Figure 10(b) due to violation of (5.6). Note that both $\underline{s}_{1}^{\omega}$ and $\underline{s}_{2}^{\omega}$ are discontinuous in this example. ${ }^{24}$

It turns out, however, that (5.11) is a necessary and sufficient condition for effective efficiency if both players' effective payoffs are higher than the static Nash levels:

Proposition 5.2. ${ }^{25}$ Let $\omega \in \mathbb{R}^{2}$ satisfy

$$
\forall i, \quad \omega_{i} \geq w_{i}\left(\hat{s}_{i}, \hat{s}_{j}\right)
$$

Suppose an IRE associated with $\omega$ exists. Then it is effectively efficient iff (5.11) holds.

Proof. See Appendix C.

For example, (5.12) trivially holds for any IRE if $\hat{s}_{i}=0$ for both $i$, as in the prisoner's dilemma game in Section 2.2.3.

\section{Applications}

\subsection{Prisoner's Dilemma}

Consider the alternating move game associated with the prisoner's dilemma in Section 2.2.3. ${ }^{26}$ For simplicity, we assume directly that the one-shot payoff of player $i$ is given by (2.4), ${ }^{27}$ and that both players have the same discount

\footnotetext{
${ }^{24}$ Though Figure 10 only shows that the IRE in (a) is "locally" effectively efficient, it should be clear that one can easily construct a fully specified example of an effectively efficient IRE that violates (5.11).

${ }^{25} \mathrm{We}$ would like to thank an anonymous referee for inspiring us to establish this result.

${ }^{26}$ The simultaneous move game can be analyzed similarly; recall Lemma 2.1 and Section 4

${ }^{27}$ Alternatively one may assume that player $i$ 's mixed action in period $t \in T_{i}$ is observable to player $j$ at the beginning of period $t+1$. In this case, player $i$ 's expected one-shot payoff in period $t$ is $-a s_{i, t}+(c+a) r_{j, t-1}$, where $s_{i, t}$ is player $i$ 's probability of choosing C, and $r_{j, t-1}$ is player $j$ 's realized action in period $t-1$. Since $r_{j, t-1}$ does not affect player $i$ 's preferences over his actions from period $t$ onward, all our results hold in this case as well. This argument is unnecessary for the simultaneous move game, where $r_{j, t-1}$ must be replaced by $s_{j, t}$.
} 


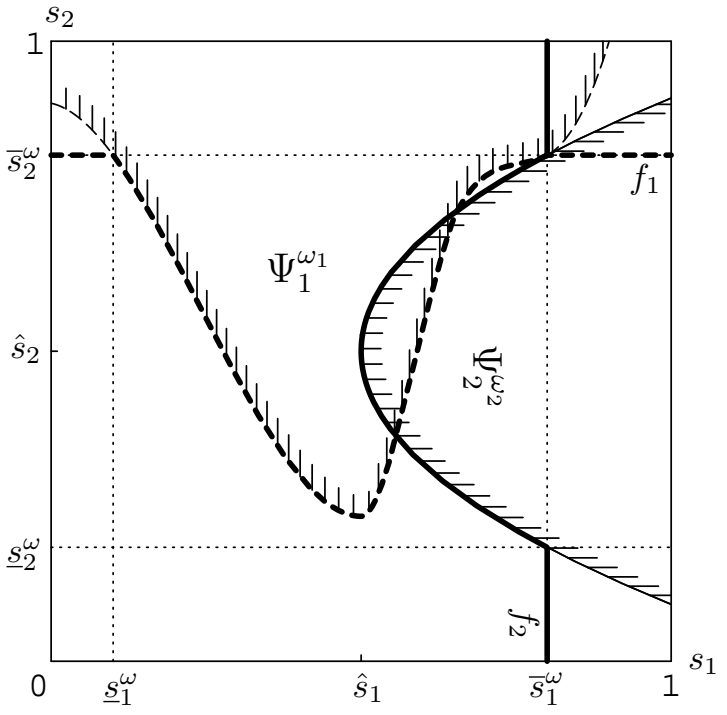

(a)

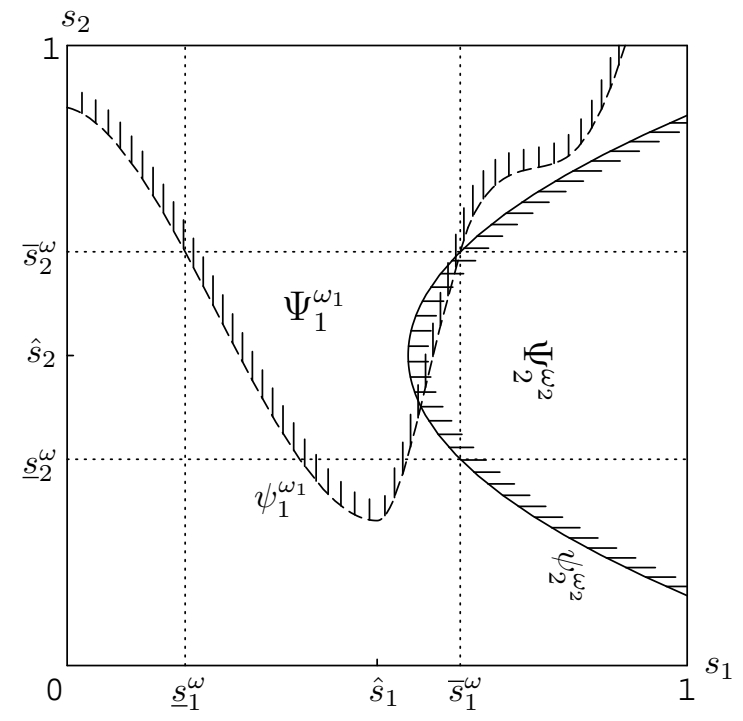

(b)

Figure 10: Effectively efficient IRE violating (5.11) 


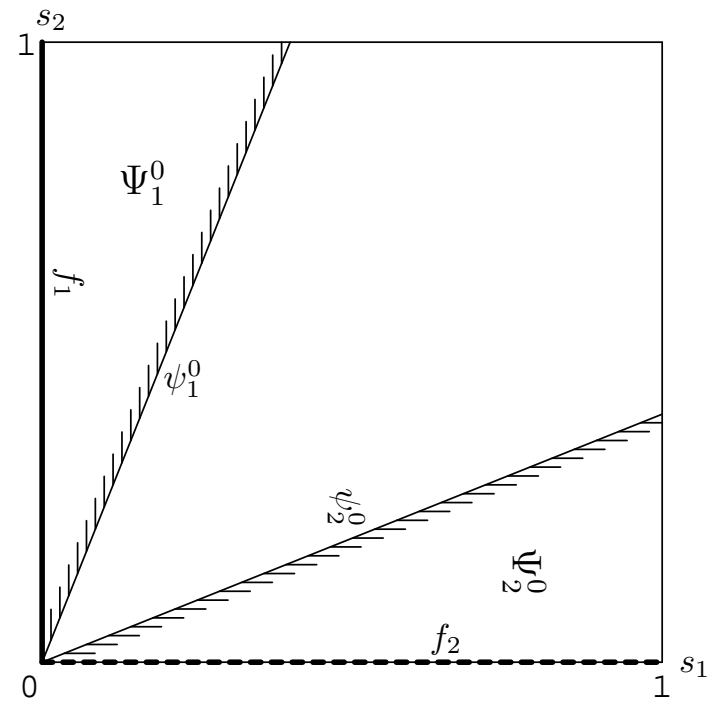

Figure 11: Case $\delta<a / e$ : Unique IRE

factor: $\delta_{1}=\delta_{2}=\delta \in(0,1)$. Player $i$ 's effective payoff is given by

$$
w_{i}\left(s_{i}, s_{j}\right)=-a s_{i}+\delta e s_{j},
$$

where $e=c+a$. Replacing $w_{i}\left(s_{i}, s_{j}\right)$ with $\omega_{i}$ and solving for $s_{j}$, we see that the indifference curve associated with $\omega^{i} \in \mathbb{R}$, or $\psi_{i}^{\omega_{i}}$, is linear:

$$
\psi_{i}^{\omega_{i}}\left(s_{i}\right)=\frac{\omega_{i}}{\delta e}+\frac{a}{\delta e} s_{i} .
$$

Since $\hat{s}_{i}=0$ here, Proposition 5.2 applies. We consider three cases separately.

Case 1: $\delta<a / e$. In this case the slope of $\psi_{i}^{\omega_{i}}$ is strictly greater than one for any $\omega \in \mathbb{R}^{2}$. By the no-sticking-out condition (5.6), $\psi_{i}^{\omega_{i}}(0) \geq \underline{s}_{j}^{\omega} \geq 0$ for both $i$ in any IRE associated with $\omega$. Thus if $\psi_{i}^{\omega_{i}}(0)>0$ for either $i$, the nonemptiness condition (5.5) is violated; see Figure 11. Hence $\psi_{i}^{\omega_{i}}(0)=0$ and $\omega_{i}=0$ for both $i$. In any IRE $\left(f_{1}, f_{2}\right)$ associated with $(0,0)$, therefore, by Proposition 5.1, $f_{i}\left(s_{j}\right) \leq \bar{s}_{i}^{(0,0)}=0$, i.e., $f_{i}\left(s_{j}\right)=0$, for all $s_{j}$ and $i$. This is the unique IRE here, which corresponds to the static Nash equilibrium; see Figure 11 again. This IRE is effectively efficient by Proposition 5.2 (or simply by uniqueness).

Case 2: $\delta=a / e$. In this knife edge case, the slope of $\psi_{i}^{\omega_{i}}$ is equal to one, and the two indifference curves emanating from the origin coincide. The 


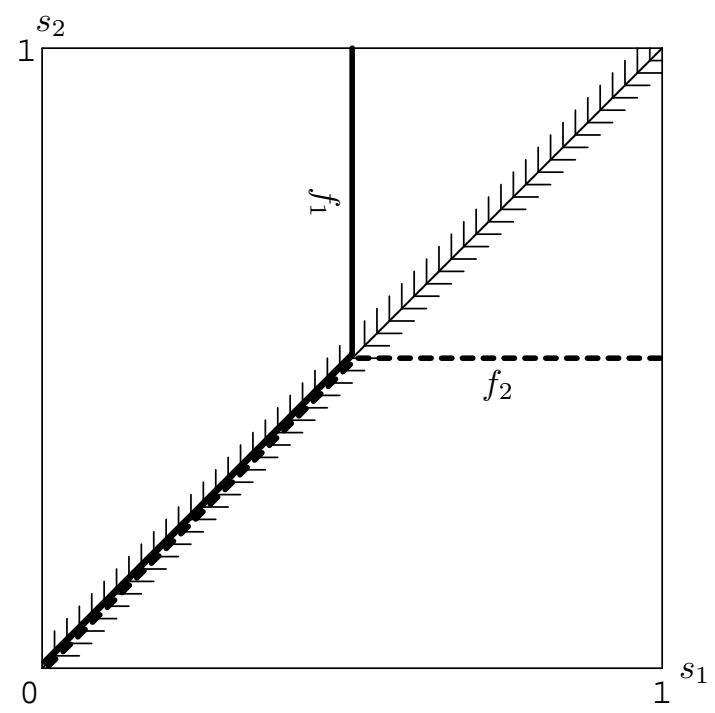

Figure 12: Case $\delta=a / e$ : Example of IRE

above argument still shows $\psi_{i}^{\omega_{i}}(0)=0$ and $\omega_{i}=0$ for both $i$. Though, as in the previous case, there is an IRE corresponding to the static Nash equilibrium, there are many other IREs here. Figure 12 depicts one example.

Case 3: $\delta>a / e$. In this case the slope of $\psi_{i}^{\omega_{i}}$ is strictly less than one, and there are many pairs of effective payoffs supported by IREs. An IRE satisfying (3.8) is depicted in Figure 13, where there is a unique and globally stable steady state. The existence of a unique and globally stable steady state is a general property of this case by (4.9) and (6.2).

Figure 14 shows a symmetric IRE that is effectively efficient. In this case, gradual cooperation occurs, and full cooperation is achieved in the long run. ${ }^{28}$ Figure 15 shows an effectively efficient IRE in which uneven gradual cooperation occurs: in the long run, only player 2 fully cooperates, while player 1 enjoys the highest possible effective payoff supported by an IRE.

\footnotetext{
${ }^{28}$ Gradual cooperation is known to arise in certain partnership games; see Furusawa and Kawakami (2008) and the references therein.
} 


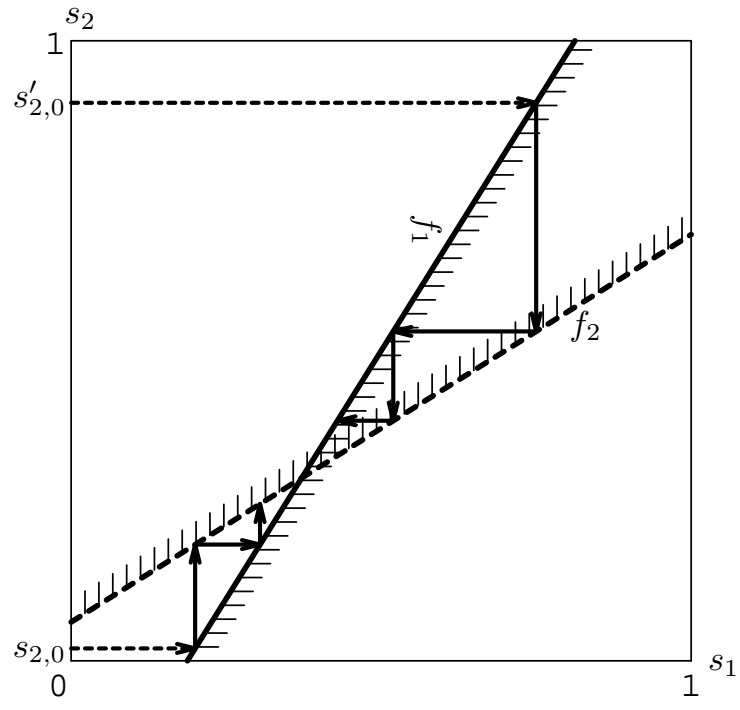

Figure 13: Case $\delta>a / e$ : "Typical" IRE

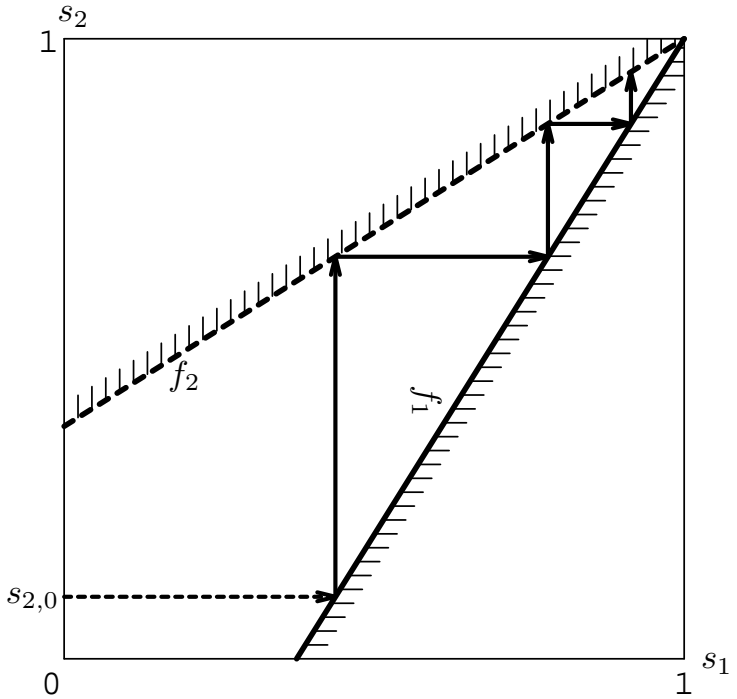

Figure 14: Case $\delta>a / e$ : Gradual cooperation 


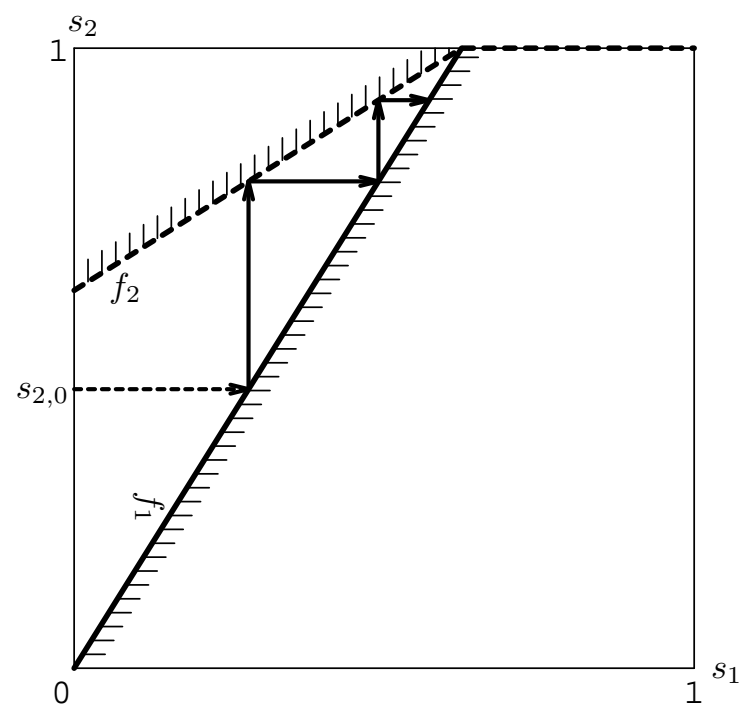

Figure 15: Case $\delta>a / e$ : Uneven gradual cooperation

\subsection{Duopoly}

Consider the alternating move game associated with the duopoly game of Section 2.2.2. ${ }^{29}$ For simplicity we assume that the firms are symmetric. Let $c$ and $\delta$ denote their common marginal cost and discount factor. Recall that firm $i$ 's one-shot profit is given by $D_{i}\left(p_{i}, p_{j}\right)\left(p_{i}-c\right)$. We parametrize $D_{i}$ as follows:

$$
D_{i}\left(p_{i}, p_{j}\right)=\left(\bar{p}-p_{i}\right) p_{j},
$$

where $\bar{p}>c$. Fix $i$. Recalling (2.2), we see that firm $i$ 's effective payoff is given by

$$
w_{i}\left(p_{i}, p_{j}\right)=\ln \left(\bar{p}-p_{i}\right)+\ln \left(p_{i}-c\right)+\delta \ln p_{j} .
$$

Replacing $w_{i}\left(p_{i}, p_{j}\right)$ with $\omega_{i}$ and solving for $p_{j}$, we obtain

$$
\psi_{i}^{\omega_{i}}\left(p_{i}\right)=\exp \left[\left\{\omega_{i}-\ln \left(\bar{p}-p_{i}\right)-\ln \left(p_{i}-c\right)\right\} / \delta\right] .
$$

Note that $\psi_{i}^{\omega_{i}}(c)=\psi_{i}^{\omega_{i}}(\bar{p})=\infty$. Direct calculation of the second derivative shows that $\psi_{i}^{\omega_{i}}$ is strictly convex. It is easy to see that given $p_{j}$, firm $i$ 's oneshot profit, as well as its effective payoff, is maximized at $p_{i}=\hat{p} \equiv(c+\bar{p}) / 2$. This is the price charged by both firms in the unique static Nash equilibrium.

\footnotetext{
${ }^{29}$ Once again, the dynamics of the simultaneous move game can be analyzed similarly.
} 


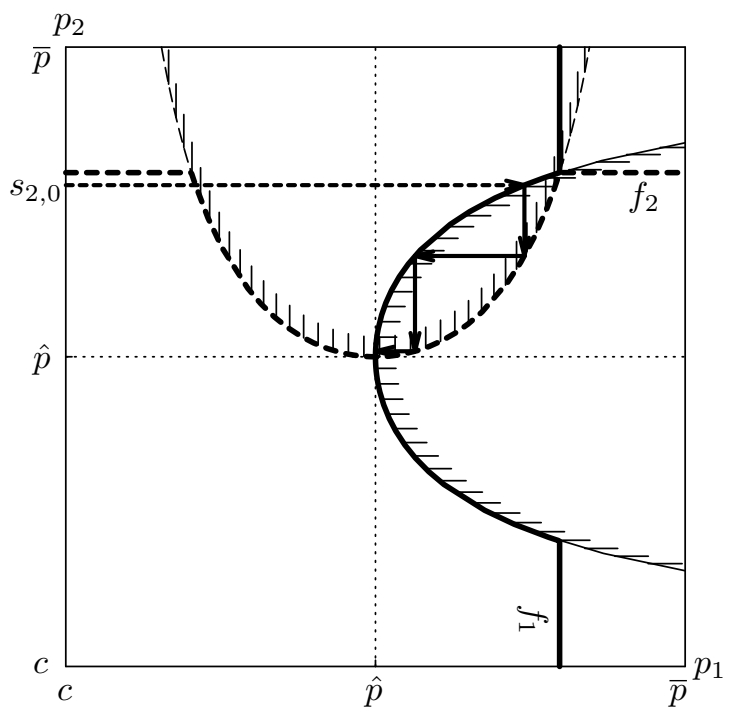

Figure 16: Kinked demand curves with unstable collusion

Figure 16 illustrates a symmetric IRE in which both firms receive the effective payoff corresponding to the static Nash equilibrium. The indifference curves in this figure are similar to those in Figure 4, which shows the IRE corresponding to the static Nash equilibrium. Figure 16 shows an alternative IRE (which is in regular form). In this IRE, there is a steady state in which both firms charge the static Nash price, as in Figure 4. In Figure 16, however, there is another steady state with a higher symmetric price. At this steady state, each firm faces a "kinked demand curve." If one of the firms raises its price, the other does not follow. Proposition 5.1 implies that this kinked feature is a rather general property in the sense that in any IRE, the firms never charge prices higher than those given by the highest intersection of the two indifference curves. On the other hand, if one of the firms lowers its price, this triggers price war, and the prices converge to the lower steady state. Figure 16 shows an example of an IRE path after a small price cut by firm 2 in period 0 (which is taken as the initial condition of the model).

Clearly the above properties of the two steady states continue to hold even if the firms receive higher effective payoffs, as long as there are two steady states. In fact there can be at most two steady states by strict concavity of $\psi_{i}^{\omega_{i}}$, provided that the firms receive effective payoffs no smaller than the static Nash level.

If there is only one steady state, then the IRE is effectively efficient by 


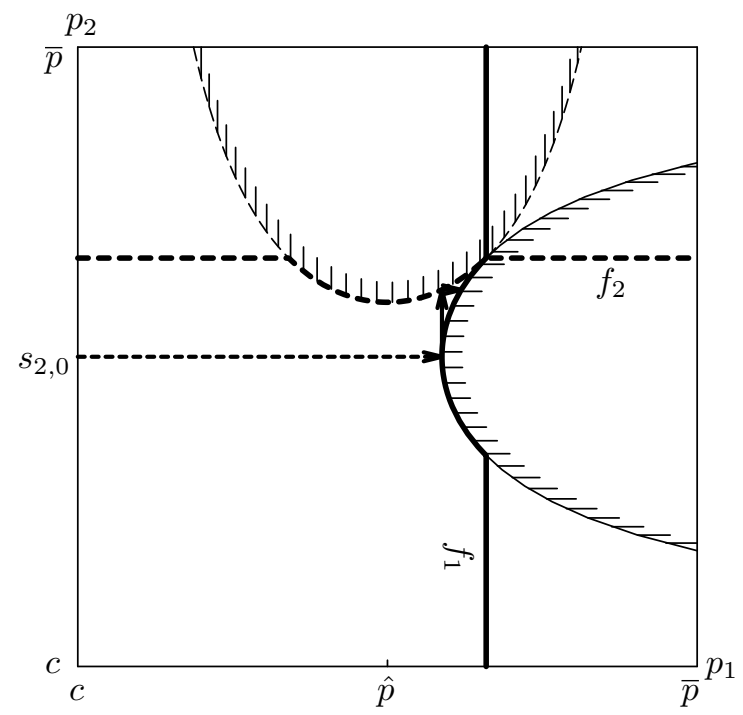

Figure 17: Kinked demand curves with stable collusion

(5.11). Figure 17 illustrates a symmetric, effectively efficient IRE in regular form. At the unique steady state, each firm faces a kinked demand curve once again. This steady state, however, is globally stable. If one of the firms raises its price, the other does not follow, as in Figure 16. If one of them lowers its price, the other lowers its price too but by a smaller degree. Eventually the prices return to the initial high level. This process is shown in Figure 17 assuming that firm 2 cuts its price to the static Nash level in period 0. It follows from Theorem 4.1 that the global stability of the unique steady state is a general property of any effectively efficient IRE here.

\section{Concluding Comments}

This paper offers a complete and graphical characterization of immediately reactive equilibria (IREs) and their global dynamics for infinitely repeated games with two players in which the action space of each player is an interval, and the one-shot payoff of each player consists of two continuous functions, one unimodal in his own action, the other strictly monotone in the opponent's action. IREs extend Nash reversion equilibria by allowing for continuous strategies and nontrivial dynamics. The global dynamics of an IRE are completely characterized by the associated indifference curves. Our 
main results show that a given pair of indifference curves is supported by an IRE if and only if it satisfies two certain graphical conditions, and that under these conditions, there is a continuous IRE that punishes deviations in a minimal way.

Although additive separability, which is crucial to our analysis, is rather restrictive, there are various interesting games that satisfy it. We have analyzed two such games and characterized their IREs by applying our general results. We have shown among other things that gradual cooperation arises in an effectively efficient IRE of a prisoners' dilemma game, and that kinked demand curves with stable collusion emerge in an effectively efficient IRE of a duopoly game.

We believe that our results are useful not only in analyzing games that satisfy our assumptions, but also in constructing completely tractable special cases of more general games. Such special cases, whose dynamics can be analyzed explicitly, would enhance the understanding of various interesting problems.

\section{Appendix A Proof of Lemma 2.1}

We reproduce (2.16) and define $M_{i}$ as follows:

$$
\forall s_{j}, \quad f_{i}\left(s_{j}\right) \in M_{i} \equiv \underset{s_{i} \in[0,1]}{\operatorname{argmax}} w_{i}\left(s_{i}, f_{j}\left(s_{i}\right)\right) .
$$

Consider the simultaneous move game. Fix $i$ and $f_{j} \in F$. From (2.13)(2.15) and (2.6), we have

$$
\sum_{t=1}^{\infty} \delta_{i}^{t-1}\left[u_{i}\left(s_{i, t}\right)+v_{i}\left(s_{j, t}\right)\right]=v_{i}\left(s_{j, 1}\right)+\sum_{t=1}^{\infty} \delta_{i}^{t-1} w_{i}\left(s_{i, t}, f_{j}\left(s_{i, t}\right)\right) .
$$

Thus the maximization problem (2.5)-(2.7) is solved iff $s_{i, t} \in M_{i}$ for all $t \in \mathbb{N}$. Hence if $f_{i} \in F$ is a best response to $f_{j}$, then $f_{i}\left(s_{j, 0}\right)=s_{i, 1} \in M_{i}$ for all $s_{j, 0}$; thus (A.1) holds. Conversely, if $f_{i} \in F$ satisfies (A.1), then $s_{i, t}=f_{i}\left(s_{j, t-1}\right) \in M_{i}$ for all $t \in \mathbb{N}$, so the maximization problem is solved, i.e., $f_{i}$ is a best response to $f_{j}$.

Now consider the alternating move game. From (2.13)-(2.15), (2.10), and 
(2.11), we have

$$
\begin{aligned}
& \sum_{t=i}^{\infty} \delta_{i}^{t-i}\left[u_{i}\left(s_{i, t}\right)+v_{i}\left(s_{j, t}\right)\right] \\
& \quad=v_{i}\left(s_{j, i}\right)+\sum_{t \in T_{i}} \delta_{i}^{t-i}\left(1+\delta_{i}\right) w_{i}\left(s_{i, t}, s_{j, t+1}\right) \\
& \quad=v_{i}\left(s_{j, i}\right)+\left(1+\delta_{i}\right) \sum_{t \in T_{i}} \delta_{i}^{t-1} w_{i}\left(s_{i, t}, f_{j}\left(s_{i, t}\right)\right) .
\end{aligned}
$$

Thus the maximization problem (2.9)-(2.11) is solved iff $s_{i, t} \in M_{i}$ for all $t \in T_{i}$. Hence the proposition follows as in the simultaneous move case.

\section{Appendix B Proof of Theorem 5.1}

We start by preparing some lemmas.

Lemma B.1. Let $\left(f_{1}, f_{2}\right) \in F^{2}$. Recall the definition of $M_{i}$ in (A.1). For $i=1,2$, we have

$$
M_{i}=\left\{s_{i} \in[0,1]\left|f_{j}\left(s_{i}\right)=\psi_{i}^{*}\right|_{f_{i}}\left(s_{i}\right)\right\} .
$$

Proof. Fix $i$. Since $w_{i}\left(s_{i}, s_{j}\right)$ is strictly increasing in $s_{j}$, we have

$$
\left.\left.w_{i}\left(s_{i}, f_{j}\left(s_{i}\right)\right)\left\{\begin{array}{c}
= \\
<
\end{array}\right\} \omega_{i}^{*}\right|_{f_{j}} \Longleftrightarrow f_{j}\left(s_{i}\right)\left\{\begin{array}{c}
= \\
<
\end{array}\right\} \psi_{i}^{*}\right|_{f_{j}}\left(s_{i}\right) .
$$

Recall Figure 3. Now (B.1) follows.

From here on we fix $\omega \in \mathbb{R}^{2}$ and omit the superscripts $\omega_{i}, \omega_{j}$, and $\omega$.

Lemma B.2. Under (5.5), for $i=1,2$,

$$
\begin{aligned}
\psi_{i}\left(\bar{s}_{i}\right) & \leq \bar{s}_{j}, \\
\psi_{i}\left(\hat{s}_{i}\right) & \leq \psi_{i}\left(\underline{s}_{i}\right) \leq \bar{s}_{j}, \\
\underline{s}_{i} & \leq \hat{s}_{i} .
\end{aligned}
$$

Proof. To see (B.3), suppose $\psi_{i}\left(\bar{s}_{i}\right)>\bar{s}_{j}$. By (5.3) and compactness of $\Psi_{i} \cap \Psi_{j}$, there is $s_{j}$ such that $\left(\bar{s}_{i}, s_{j}\right) \in \Psi_{i} \cap \Psi_{j}$. By $(5.2), \psi_{i}\left(\bar{s}_{i}\right) \leq s_{j}$. But then $\bar{s}_{j}<\psi_{i}\left(\bar{s}_{i}\right) \leq s_{j}$, contradicting the definition of $\bar{s}_{j}$.

Note from (5.4) that $\psi_{i}\left(\underline{s}_{i}\right) \leq \bar{s}_{j}$. We obtain (B.4) since $\psi_{i}\left(s_{i}\right)$ is minimized at $s_{i}=\hat{s}_{i}($ recall (3.2)). Finally (B.5) follows from (B.4) and (5.4). 
The sufficiency part of Theorem 5.1 follows from Theorem 5.2, which we prove first.

\section{B.1 Sufficiency: Proof of Theorem 5.2}

Assume (5.5) and (5.6). Define $\left(f_{1}, f_{2}\right)$ by (5.7). To show that $\left(f_{1}, f_{2}\right)$ is an IRE, it suffices to verify (A.1) for $i=1,2$ by Lemma 2.1. Fix $i$. By (B.4) and (5.7) (with $i$ and $j$ interchanged),

$$
\forall s_{i}, \quad f_{j}\left(\hat{s}_{i}\right)=\psi_{i}\left(\hat{s}_{i}\right) \leq f_{j}\left(s_{i}\right) \leq \psi_{i}\left(s_{i}\right)
$$

where the first inequality holds since $\psi_{i}\left(s_{i}\right)$ is minimized at $s_{i}=\hat{s}_{i}$ (recall $(3.2))$. Since $w_{i}\left(s_{i}, s_{j}\right)$ is increasing in $s_{j}$, it follows that

$$
\begin{aligned}
\forall s_{i}, \quad w_{i}\left(s_{i}, f_{j}\left(s_{i}\right)\right) & \leq w_{i}\left(s_{i}, \psi_{i}\left(s_{i}\right)\right)=\omega_{i} \\
& =w_{i}\left(\hat{s}_{i}, \psi_{i}\left(\hat{s}_{i}\right)\right)=w_{i}\left(\hat{s}_{i}, f_{j}\left(\hat{s}_{i}\right)\right) .
\end{aligned}
$$

This implies $\left.\omega_{i}^{*}\right|_{f_{j}}=\omega_{i}$ and $\left.\psi_{i}^{*}\right|_{f_{j}}=\psi_{i}$. Hence by (B.1) and (5.7),

$$
M_{i}=\left\{s_{i} \in[0,1] \mid \psi_{i}\left(s_{i}\right) \leq \bar{s}_{j}\right\} .
$$

It follows by (B.3) and (B.4) that $\underline{s}_{i}, \bar{s}_{i} \in M_{i}$. Fix $s_{j}$. We have

$$
\underline{s}_{i} \leq \psi_{j}\left(\hat{s}_{j}\right) \leq f_{i}\left(s_{j}\right) \leq \bar{s}_{i}
$$

by (5.6), (B.6) (with $i$ and $j$ interchanged), and (5.7). Since $\underline{s}_{i}, \bar{s}_{i} \in M_{i}$ and $M_{i}$ is an interval by (B.9), we obtain $f_{i}\left(s_{j}\right) \in M_{i}$, i.e., (A.1).

Regarding (5.8), since $f_{i}\left(f_{j}(\cdot)\right)$ is a continuous function from $[0,1]$ to itself, it has a fixed point $s_{i}$ (i.e., $\left.s_{i}=f_{i}\left(f_{j}\left(s_{i}\right)\right)\right)$ by Brouwer's fixed point theorem. Let $s_{j}=f_{j}\left(s_{i}\right)$. Then $\left(s_{1}, s_{2}\right)$ is a steady state and satisfies (5.8)(b) by Theorem 4.1. This in turn implies (5.8)(a).

\section{B.2 Necessity}

Let $\left(f_{1}, f_{2}\right)$ be an IRE associated with $\omega$. We wish to verify (5.5) and (5.6). Although (B.3) implies $\left(\bar{s}_{1}, \bar{s}_{2}\right) \in \Psi_{1} \cap \Psi_{2}$, we cannot show $\Psi_{1} \cap \Psi_{2} \neq \emptyset$ by using $\left(\bar{s}_{1}, \bar{s}_{2}\right)$, whose existence requires $\Psi_{1} \cap \Psi_{2} \neq \emptyset$. Thus we need to find an action profile defined in terms of $\left(f_{1}, f_{2}\right)$ that belongs to $\Psi_{1} \cap \Psi_{2}$.

To this end, note that since $\left(f_{1}, f_{2}\right)$ is an IRE associated with $\omega$, for $i=1,2$,

$$
\psi_{i}\left(=\psi_{i}^{\omega_{i}}\right)=\left.\psi_{i}^{*}\right|_{f_{j}}
$$


Define

$$
\begin{aligned}
D_{i} & =\left\{s_{i} \in[0,1] \mid \psi_{i}\left(s_{i}\right) \in[0,1]\right\}, \\
R_{i} & =\left\{s_{i} \in[0,1] \mid \exists s_{j} \in[0,1], s_{i}=f_{i}\left(s_{j}\right)\right\} \\
\bar{r}_{i} & =\sup R_{i}, \\
\underline{r}_{i} & =\min \left\{s_{i} \in D_{i} \mid \psi_{i}\left(s_{i}\right) \leq \bar{r}_{j}\right\} .
\end{aligned}
$$

Note that $D_{i}$ is the domain of $\psi_{i}$ when its range is restricted to $[0,1], R_{i}$ is the range of $f_{i}$, and $\underline{r}_{i}$ exists since there is $s_{i}$ with $f_{j}\left(s_{i}\right)=\psi_{i}\left(s_{i}\right)$ by (B.1) and (B.11). The idea here is to use $\underline{r}_{i}$ and $\bar{r}_{i}$ as substitutes for $\underline{s}_{i}$ and $\bar{s}_{i}$.

Lemma B.3. For $i=1,2$, we have

$$
\text { (a) } \bar{r}_{i} \in D_{i}, \quad \text { (b) } \psi_{i}\left(\bar{r}_{i}\right) \in D_{j}, \quad(\mathrm{c}) \psi_{j}\left(\psi_{i}\left(\bar{r}_{i}\right)\right) \leq \bar{r}_{i} \text {. }
$$

Proof. Fix $i$. By (A.1) (which holds by Lemma 2.1), (B.1), and (B.11),

$$
R_{i} \subset M_{i} \subset D_{i}
$$

Since $D_{i}$ is compact by continuity of $\psi_{i},{ }^{30}$ (a) follows from (B.17).

Note from (3.6), (B.11), and (B.17) (with $i$ and $j$ interchanged) that

$$
\forall s_{i} \in R_{i}, \quad \psi_{i}\left(s_{i}\right)=f_{j}\left(s_{i}\right) \in R_{j} \subset D_{j}
$$

Thus (b) follows by continuity of $\psi_{i}$ and compactness of $D_{j}$.

For (c), note from (B.18) (with $i$ and $j$ interchanged) that $\sup _{s_{j} \in R_{j}} \psi_{j}\left(s_{j}\right) \leq$ $\bar{r}_{i}$. Since $\psi_{i}\left(s_{i}\right) \in R_{j}$ for $s_{i} \in R_{i}$ by (B.18),

$$
\forall s_{i}, \quad \psi_{j}\left(\psi_{i}\left(s_{i}\right)\right) \leq \sup _{s_{j} \in R_{j}} \psi_{j}\left(s_{j}\right) \leq \bar{r}_{i}
$$

Now (c) follows by continuity of $\psi_{i}$ and $\psi_{j}$.

Lemma B.4. For $i=1,2$,

$$
\begin{gathered}
\left(\bar{r}_{i}, \psi_{i}\left(\bar{r}_{i}\right)\right) \in \Psi_{i} \cap \Psi_{j}, \\
\text { (a) } \hat{s}_{j} \in D_{j}, \quad \text { (b) } \underline{r}_{i} \leq \psi_{j}\left(\hat{s}_{j}\right) .
\end{gathered}
$$

\footnotetext{
${ }^{30}$ Note that $R_{i}$ need not be compact since $f_{i}$ need not be continuous.
} 
Proof. Fix $i$. We have $\left(\bar{r}_{i}, \psi_{i}\left(\bar{r}_{i}\right)\right) \in \Psi_{i}$ by (5.2) (with $s_{i}=\bar{r}_{i}$ and $s_{j}=$ $\psi_{i}\left(\bar{r}_{i}\right)$ ) and (B.16)(a),(b). By (5.2) (with $i$ and $j$ interchanged) and (B.16), $\left(\bar{r}_{i}, \psi_{i}\left(\bar{r}_{i}\right)\right) \in \Psi_{j} .{ }^{31}$ Thus (B.20) follows.

For (B.21), note from (B.2) and (B.11) that

$$
0 \leq f_{i}\left(\hat{s}_{j}\right) \leq \psi_{j}\left(\hat{s}_{j}\right) \leq 1,
$$

which implies (B.21)(a). By (B.1), (B.11), and (B.14),

$$
\forall s_{i} \in M_{i}, \quad \psi_{i}\left(s_{i}\right)=f_{j}\left(s_{i}\right) \leq \bar{r}_{j} .
$$

Hence $M_{i} \subset\left\{s_{i} \in D_{i} \mid \psi_{i}\left(s_{i}\right) \leq \bar{r}_{j}\right\} \equiv A_{i}$. Thus by (B.22) and (A.1),

$$
\psi_{j}\left(\hat{s}_{j}\right) \geq f_{i}\left(\hat{s}_{j}\right) \in M_{i} \subset A_{i} .
$$

Note from (B.15) that

$$
\underline{r}_{i}=\min A_{i} .
$$

Thus (B.21)(b) follows.

Let us now complete the proof of Theorem 5.1. We have (5.5) by (B.20). Fix $i$. By (B.20) and (5.3) (both with $i$ and $j$ interchanged),

$$
\bar{r}_{j} \leq \bar{s}_{j} .
$$

Thus $A_{i} \subset\left\{s_{i} \in[0,1] \mid \psi_{i}\left(s_{i}\right) \leq \bar{s}_{j}\right\}$. Recalling (B.25) and (5.4), we have $\underline{r}_{j} \geq \underline{s}_{j}$. This together with (B.21) establishes (5.6).

\section{Appendix C Proof of Proposition 5.2}

Sufficiency follows from the discussion leading to (5.11). For necessity, fix an $\operatorname{IRE}\left(f_{1}, f_{2}\right)$ associated with $\omega \in \mathbb{R}^{2}$ satisfying (5.12). This implies

$$
\forall i, \quad \psi_{i}^{\omega_{i}}\left(\hat{s}_{i}\right) \geq \hat{s}_{j} .
$$

Suppose (5.11) does not hold, i.e., $\tilde{\Psi}_{1}^{\omega_{1}} \cap \tilde{\Psi}_{2}^{\omega_{2}} \neq \emptyset$. Since each $\psi_{i}^{\omega_{i}}$ is continuous and strictly increasing in $\omega_{i}$ by (3.2), there is $\omega^{\prime} \gg \omega$ such that $\tilde{\Psi}_{1}^{\omega_{1}^{\prime}} \cap \tilde{\Psi}_{2}^{\omega_{2}^{\prime}} \neq \emptyset$ and $\psi_{i}^{\omega_{i}^{\prime}}\left(\hat{s}_{i}\right)>\psi_{i}^{\omega_{i}}\left(\hat{s}_{i}\right)$ for $i=1,2$. This together with (C.1) and (B.5) yields $\psi_{i}^{\omega_{i}^{\prime}}\left(\hat{s}_{i}\right)>\psi_{i}^{\omega_{i}}\left(\hat{s}_{i}\right) \geq \hat{s}_{j} \geq \underline{s}_{i}^{\omega^{\prime}}$, which implies (5.6). Thus by Theorem 5.1, there is an IRE associated with $\omega^{\prime}$, so $\left(f_{1}, f_{2}\right)$ cannot be effective efficient.

\footnotetext{
${ }^{31}$ Recall footnote 20 .
} 


\section{References}

Bhaskar, V., S. Machin, G. Reid, 1991, Testing a model of the kinked demand curve, Journal of Industrial Economics 39, 241-154.

Devaney, R.L., 1989, An Introduction to Chaotic Dynamical Systems, 2nd ed. Redwood City: Addison-Wesley.

Figuières, C., Jean-Marie, A., Quérou, N., Tidball, M., 2004, Theory of Conjectural Variations. New Jersey: World Scientific.

Friedman, J.W., 1968, Reaction functions and the theory of duopoly, Review of Economic Studies 35, 257-272.

Friedman, J.W., 1971, A non-cooperative equilibrium for supergames, Review of Economic Studies 38, 1-12.

Friedman, J.W., L. Samuelson, 1990, Subgame perfect equilibria with continuous reaction functions, Games and Economic Behavior 2, 304-324.

Friedman, J.W., L. Samuelson, 1994a, Continuous reaction functions in duopolies, Games and Economic Behavior 6, 55-82.

Friedman, J.W., L. Samuelson, 1994b, An extension of the "folk theorem" with continuous reaction functions, Games and Economic Behavior 6, $83-96$.

Fudenberg, D., J. Tirole, 1991, Game Theory. Cambridge, MA: MIT Press.

Furusawa, T., T. Kamihigashi, 2006, Threats and concessions in tariff setting, work in progress.

Furusawa, T., T. Kawakami, 2008, Gradual cooperation in the existence of outside options, Journal of Economic Behavior and Organization 68, 378389.

Haller, H., R. Lagunoff, 2000, Markov perfect equilibria in repeated asynchronous choice games, working paper, Georgetown University.

Kalai, E., D. Samet, W. Standford, 1988, A note on reactive equilibria in the discounted prisoner's dilemma and associated games, International Journal of Game Theory 17, 177-186.

Kamihigashi, K., S. Roy, 2006, Dynamic optimization with a nonsmooth, nonconvex technology: the case of a linear objective function, Economic Theory 29, 325-340.

Kranton, R.E., 1996, The formation of cooperative relationships, Journal of Law, Economics, and Organization 12, 214-233. 
Langlois, J.-P., J.A. Sachs, 1993, Existence and local stability of Pareto superior reaction function equilibria in discounted supergames, Journal of Mathematical Economics 22, 199-221.

Lagunoff, R., A. Matsui, 1997, Asynchronous choice in repeated coordination games, Econometrica 65, 1467-1477.

Maskin, E., J. Tirole, 1988a, A theory of dynamic oligopoly, I: overview and quantity competition with large fixed costs, Econometrica 56, 549-569.

Maskin, E., J. Tirole, 1988b, A theory of dynamic oligopoly, II: price competition, kinked demand curves, and edgeworth cycles, Econometrica 56, $571-599$.

Maskin, E., J. Tirole, 2001, Markov perfect equilibrium I: observable actions, Journal of Economic Theory 100, 191-291.

Radner, R., 2003, Viscous demand, Journal of Economic Theory 112, 189 231.

Rand, D., 1978, Exotic phenomena in games and duopoly models, Journal of Mathematical Economics 5, 173-184.

Robson, A., 1986, The existence of Nash equilibria in reaction functions for dynamic models of oligopoly, International Economic Review 27, 539544.

Rosser, J.B., Jr., 2002, The development of complex oligopoly dynamics theory. In: T. Puu, I. Sushko, (Eds.), Oligopoly Dynamics: Models and Tools, Springer-Verlag, Berlin, pp. 15-29.

Sabourian, H., 1992, Rational conjectural equilibrium and repeated games. In: P. Dasgupta, D. Gale, O. Hart, E. Maskin, (Eds.), Economic Analysis of Markets and Games: Essays in Honor of Frank Hahn, MIT Press, pp. $228-257$.

Samuelson, L., 1987, Non-trivial subgame perfect duopoly equilibria can be supported by continuous reaction functions, Economics Letters 24, 207211.

Sen, D., 2004, The kinked demand curve revisited, Economics Letters 84, 99-105.

Stanford, W.G., 1986, Subgame perfect reaction function equilibria in discounted duopoly supergames are trivial, Journal of Economic Theory 39, $226-232$.

Yoon, K., 2001, A folk theorem for asynchronously repeated games, Econometrica 69, 191-200. 
Watson, J., 1999, Starting small and renegotiation, Journal of Economic Theory 85, 52-90.

Watson, J., 2002, Starting small and commitment, Games and Economic Behavior 38, 176-199. 